\title{
Design and use of model membranes to study biomolecular interactions using complementary surface- sensitive techniques
}

\author{
Luke A. Clifton, Richard A. Campbell, Federica Sebastiani, José \\ Campos-Terán, Juan F. Gonzalez-Martinez, Sebastian Björklund, \\ Javier Sotres and Marité Cárdenas
}

\section{Published version information}

Citation: LA Clifton et al. "Design and use of model membranes to study biomolecular interactions using complementary surface-sensitive techniques." Advances in Colloid and Interface Science, vol. 277 (2020): 102118.

(C)2020. This manuscript version is made available under the CC-BY-NC-ND 4.0 Licence.

This version is made available in accordance with publisher policies. Please cite only the published version using the reference above. This is the citation assigned by the publisher at the time of issuing the AAM. Please check the publisher's website for any updates. 


\section{Design and Use of Model Biomembranes to Study Biomolecular Interactions using Complementary Surface-Sensitive Techniques}

Luke A. Clifton ${ }^{1}$, Richard A. Campbell2, Federica Sebastiani ${ }^{3}$, José Campos-Terán ${ }^{4,5}$, Juan F. Gonzalez-Martinez ${ }^{3}$, Sebastian Björklund ${ }^{3}$, Javier Sotres ${ }^{3}$ and Marité Cárdenas ${ }^{3}$

${ }^{1}$ ISIS Pulsed Neutron and Muon Source, Science and Technology Facilities Council, Rutherford Appleton Laboratory, Harwell Science and Innovation Campus, Didcot, Oxfordshire, OX11 OQX, UK.

${ }^{2}$ Division of Pharmacy and Optometry, University of Manchester, Manchester M13 9PT, United Kingdom.

${ }^{3}$ Department of Biomedical Science and Biofilms - Research Center for Biointerfaces, Malmö University, 20506 Malmö, Sweden.

${ }^{4}$ Departamento de Procesos y Tecnología, Universidad Autónoma Metropolitana, Unidad Cuajimalpa, Av. Vasco de Quiroga 4871,Col. Santa Fe, Delegación Cuajimalpa de Morelos 05348, Mexico

${ }^{5}$ Lund Institute of advanced Neutron and X-ray Science, Lund University, Scheelevägen 19, 22370 Lund, Sweden

Author contribution statement: L.A.C, R.A.C., J.S. \& M.C: conceptualization; All: data curation; All: formal analysis; M.C.: funding acquisition; All: writing - original draft, review \& editing.

Five keywords for indexing: biomembranes, biomolecular interactions, surface-sensitive techniques, lipids, antimicrobial peptides. 


\section{Highlights}

- Model biomembranes at surfaces are useful to study biomolecular interactions

- The model membrane complexity can be adapted from one to multiple components

- Implementations range from lipid monolayers to a range of supported bilayers

- Complementary surface-sensitive techniques allow studying model membranes

- Information accessible is reviewed via the use of the peptide Melittin 


\begin{abstract}
Cellular membranes are complex structures and simplified analogues, or "model membranes" or "biomembranes", are used as platforms to understand fundamental properties of the membrane itself as well as interactions with various biomolecules such as drugs, peptides and proteins. Model membranes at the air-liquid and solid-liquid interfaces can be studied using a range of complementary surface-sensitive techniques to give a detailed picture of both the structure and physicochemical properties of the membrane and its resulting interactions. In this review, we will present the main planar model membranes used in the field to date with a focus on monolayers at the air-liquid interface, supported lipid bilayers at the solid-liquid interface and advanced membrane models such as tethered and floating membranes. We will then briefly present the principles as well as the main type of information on molecular interactions at model membranes accessible using a Langmuir trough, quartz crystal microbalance with dissipation monitoring, ellipsometry, atomic force microscopy, Brewster angle microscopy, Infrared spectroscopy, neutron and X-ray reflectometry. A consistent example for following biomolecular interactions at model membranes is used across many of the techniques in terms of the well-studied antimicrobial peptide Melittin. The overall objective is to establish an understanding of the information accessible from each technique, their respective advantages and limitations, and their complementarity.
\end{abstract}




\section{Graphical abstract}

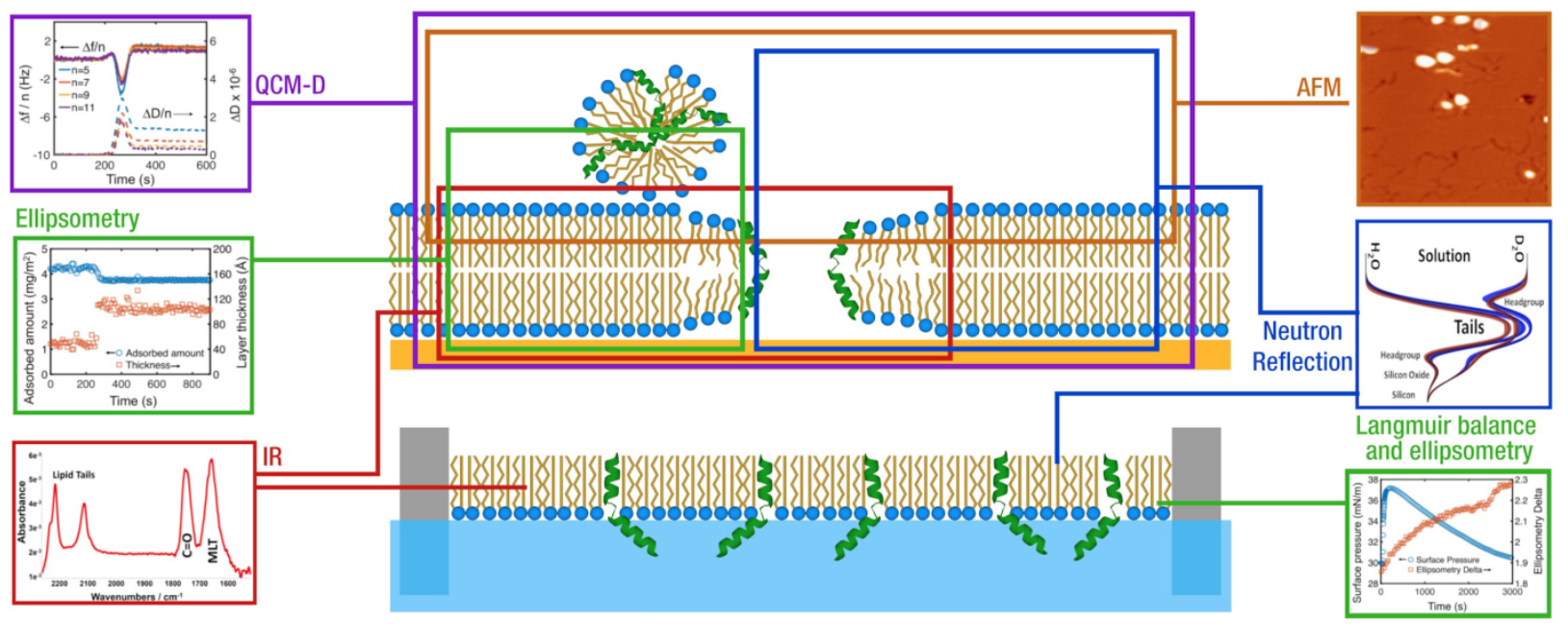




\section{Contents of the paper}

1. Scope

2. Introduction

3. Membrane Architecture and Lipid Composition of Bacterial and Red Blood Cells

3.1 The Fluid Mosaic Model

3.2 The Membrane Architecture of Prokaryotes and Eukaryotes

4. Models of Cellular Membranes at Surfaces

4.1 Lipid Monolayers at the Air-Liquid Interface

4.2 Supported Lipid Bilayers at the Solid-Liquid Interface

4.3 Advanced Planar Membrane Systems

5. Surface-sensitive Techniques to study Molecular Interactions at Model Biomembranes

5.1 Changes in Interfacial Mass from Quartz Crystal Microbalance with Dissipation Monitoring and Ellipsometry

5.2 Imaging Interfacial Morphologies using Atomic Force Microscopy and Brewster Angle Microscopy

5.3 Determination of Interfacial Interactions with Specific Chemical Groups Using Infrared Spectroscopy

5.4 Resolution of the Interfacial Structure and Composition by Neutron Reflectometry and X-ray Reflectometry

6. Complementarity of Surface-Sensitive Techniques to reveal the Interaction Mechanisms of an Antimicrobial Peptide with Model Biomembranes

6.1 Complementary Comparison of Analytical Data

6.2 Critical Comparison of Analytical Techniques

6.3 The relevance of the lipids choice and experimental conditions

7. Perspectives and Conclusions 


\section{Scope}

In this review, we present an overview of the main types of model cellular membranes (biomembranes) that can be used in combination with various surface-sensitive techniques to gain an understanding of intermolecular interactions. Relevant interactions include drug effects on membrane architecture, protein-membrane binding and the action of antimicrobial compounds such a natural or synthetic peptides, to name a few. While there are several earlier reviews on this general topic (to name a few recent examples see refs [1-4]), this review provides uniquely both an overview of considerations to take into account in the design of the platform and composition of a model cellular membrane system, and a critical discussion of the advantages and limitations of different surfacesensitive techniques to obtain information about resulting interactions with biomolecules of interest.

We will start by reviewing the main cellular architectures and the lipid compositions typically mimicked in biomembrane models. Then we will discuss the main models that are used including monolayers at the air-liquid interface, supported lipid bilayers and advanced planar membrane models such as tethered or floating lipid bilayers at the solidliquid interface. Lastly, we will detail the use of a number of analytical techniques used to study planar model cellular membranes and, through the use of a consistent example, give a brief overview of each technique and the information accessible.

The aim of this approach is for the reader to gain an understanding of the value of each technique in its application to biomembrane studies and, ultimately, how to combine techniques to gain a detailed understanding of membrane related phenomena through complementary comparison on the molecular level. The importance of the choice of model membrane composition and experimental conditions when assessing intermolecular interactions will be considered throughout the discussion.

The biomolecular interaction used as an example is of the cationic antimicrobial peptide Melittin with model lipid membranes that bear a net negative charge. This example was chosen due to the prevalence in the literature of studies on this peptide combined with its strong disruptive effect on membrane mimetics. Nevertheless, experimental conditions in such publications across different model membrane platforms vary widely, which 
motivated the collection of data recorded under similar experimental conditions using multiple experimental techniques to aid a coherent comparison. An overview of the advantages and limitations of each technique in relation to biomembrane studies, as well as a perspective on the complementarity of their combined application, is delivered.

\section{Introduction}

Cellular membranes are key cellular components that define biology in many senses. On the one hand, this complex material designates what is and is not inside of the cell. On the other hand, it constitutes the many organelles inside the cell that carry out cellular function. Composed of a bilayer of lipids with embedded and associated proteins, cellular membranes are involved in almost every biochemical process (Figure 1). It is therefore not surprising that cellular membranes are the target for $\sim 60 \%$ of drugs $[5,6]$.

Cellular membranes are highly complex in terms of composition while having a small transverse size of barely 6 nanometers. Therefore, it is challenging to gain precision molecular level information on membranes and their constituents in living organisms (i.e. in vivo). In this respect, the development of planar membrane models (or planar biomembranes) was an asset to the study of membrane biophysics. Initially consisting of lipid monolayers at the air-liquid interface or lipid bilayers deposited at the solid-liquid interface, a series of increasingly complex and biologically accurate model architectures have now been developed.

Analytical techniques which operate at bulk interfaces are commonly employed in the research environment. Whether it is atomic force microscopy (which uses the interaction of a probe directly with a solid support) or reflectometry measurements (which measure interference patterns in surface reflected waves to examine structure), many techniques rely on some beneficiary facet(s) of the interface between two phases of matter to operate. Planar model membrane systems by their very nature sit at or close to a bulk interface, and therefore are applicable to study by surface-sensitive techniques. The study of model membranes using bench-top surface analysis techniques such as quartz crystal microbalance with dissipation monitoring (QCM-D), ellipsometry, atomic force microscopy (AFM), Brewster angle microscopy (BAM) and different forms of Infrared 
spectroscopy, as well as large scale facility techniques such as neutron reflectometry $(\mathrm{NR})$ and X-ray reflectometry (XRR), can provide a precision understanding of membrane-relevant events using mimetic sample systems with ever increasing biological accuracy. A combination of multiple complementary measurements with biologically accurate samples is key in these studies if a realistic understanding of membrane related phenomena is to be revealed in these studies. This review act as a guide to this approach in order to provide the reader with information to aid the design of future biomembranes studies.

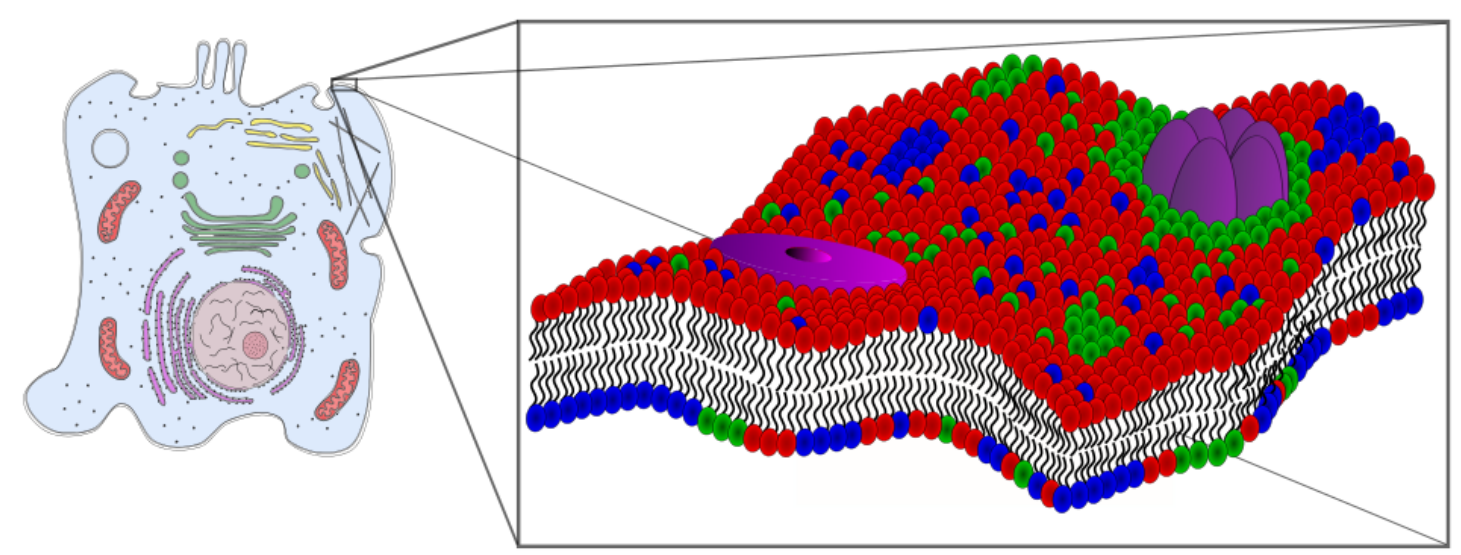

Figure 1. The Fluid Mosaic Model of a Biological Membrane, with focus on plasma membranes.

\section{Membrane Architecture and Lipid Composition for Bacterial and Red Blood Cells}

\subsection{The Fluid Mosaic Model}

In 1972, Singer and Nicolson proposed the "fluid mosaic" membrane model [7]: the cell membrane is represented by a viscous, though fluid, lipid bilayer in which membrane proteins are embedded and free to diffuse within the lipid bilayer plane. The fluidity of cellular membranes is ensured by the presence of mono- and poly- unsaturated fatty acid chains in lipids [8] as well as the presence of cyclic propyl groups in bacterial cells $[9,10]$. However, most cells present a range of saturated, long chain fatty acids that give rise to non-fluid membranes besides high melting temperature lipids such as sphingolipids and sterols [8]. The presence of fluid and non-fluid lipids gives rise to lipid-lipid interactions 
that were not considered in the fluid mosaic model from 1972. Israelachvili modified the fluid mosaic model in 1977 to include thermodynamic and molecular packing factors to describe the highly coupled organization of lipids and proteins [11] due to lipid-protein and lipid-lipid interactions. But it wasn't until 2014 that Nicholson incorporated the effects of non-random intermolecular interactions between the membrane component into the fluid mosaic model [12]. One of the properties arising from specific lipid-lipid and lipidprotein associations is the formation of ordered and specialized "raft-like"membrane domains, which are proposed to be highly dynamic and fluctuating nano-scaled assemblies [9].

\subsection{The Membrane Architecture of Prokaryotes and Eukaryotes.}

Within the scope of the general structure of biomembranes presented by the fluid mosaic model, there are major differences in membrane architectures between cell types. The architecture of the plasma membrane differs between eukaryotic and prokaryotic cells as shown in Figure 2 for bacteria and red blood cells (erythrocytes). Model bacterial membranes are commonly used to examine the interaction of antimicrobial compounds [13-16], while model erythrocyte membranes are used to examine cytotoxicity and drug interactions $[17,18]$.

Bacterial species are classed as either Gram negative or Gram positive based on differences in their surface architectures (which give rise to differential absorption of the Gram stain). Gram negative bacteria possess two membranes: the inner membrane is situated between the cytoplasm and periplasm, while the outer membrane is located between the periplasm and the extracellular environment. The inner membrane is predominantly composed of membrane bound or associated proteins and the phospholipids phosphatidylglycerol (PG), phosphaphatidylethanolamine (PE) and cardiolipin (CL) [10]; see Figure 2. The Gram negative bacterial outer membrane is asymmetric in terms of its lipid components: the inner leaflet of the outer membrane is composed of similar phospholipids to the inner membrane while the outer leaflet is composed entirely of liposaccharide (LPS) molecules $[19,20]$. These complex glycolipids face the extracellular environment via long polysaccharide chains which protect the 
bacteria from harmful agents such as hydrophobic antibiotics. They are negatively charged predominantly due to phosphate groups located in the core oligosaccharide region and are stabilized by divalent cations which bridge these groups between neighbouring LPS molecules.

Conversely, Gram positive bacteria possess a single membrane. It is predominantly composed of the anionic phospholipids PG and CL. Adjacent to the membrane, and facing the extracellular environment, are multilayers of peptidoglycan with a total thickness up to $80 \mathrm{~nm}$. Peptidoglycan is composed of chains of alternating amino sugars, with individual polysaccharide chain linked via short polypeptides. Each layer of peptidoglycan forms a net around the bacterium. This layer acts to protect the microbe from osmotic stress and additionally prevents molecules less than $\sim 2 \mathrm{~nm}$ in size from entering the cell [21].

Mammalian cell membranes are supported by the cytoskeleton, which gives the cell mechanical stability. Red blood cells are unusual mammalian cells, as they lack an actinbased cytoskeleton and instead have a spectrin-based support that is flexible and allows red blood cells to adapt different conformations to be able to move through the narrow blood capillaries [22]. The lipid composition of eukaryotic membranes is dramatically different from that of bacterial cells and varies significantly within cell types [23]. In general, mammalian cell membranes are mainly composed of the zwitterionic phosphatidylcholine (PC) and contain minor quantities of the zwitterionic PE, the negatively charged phosphatidylserine (PS), the negative phosphatidylinositols ( $\mathrm{PI}$ ), sphingolipids (SM) and cholesterol. The cholesterol content can vary from a few \% in endothelial cells [24] and red blood cells [25] to up to $40 \%$ in brain cells [26]. With regards to the fatty acid species, mammalian cells are particularly enriched with polyunsaturated (2 or more double bonds) and longer acyl chains (> C16) [8]. Lastly, lipid asymmetry is very important in animal cells due to a combination of lipid-lipid interactions [27-29] and the function of specific, energy consuming transmembrane proteins called flipases [30]. Indeed, the loss of lipid asymmetry typically signals cell death among other biological processes [31]. Figure 2 gives the lipid composition for red blood cells. As already mentioned, besides the plasma membrane, mammalian cells contain various internal 
organelles that present highly specialized cellular membranes to keep the organelles isolated from the cytosol.

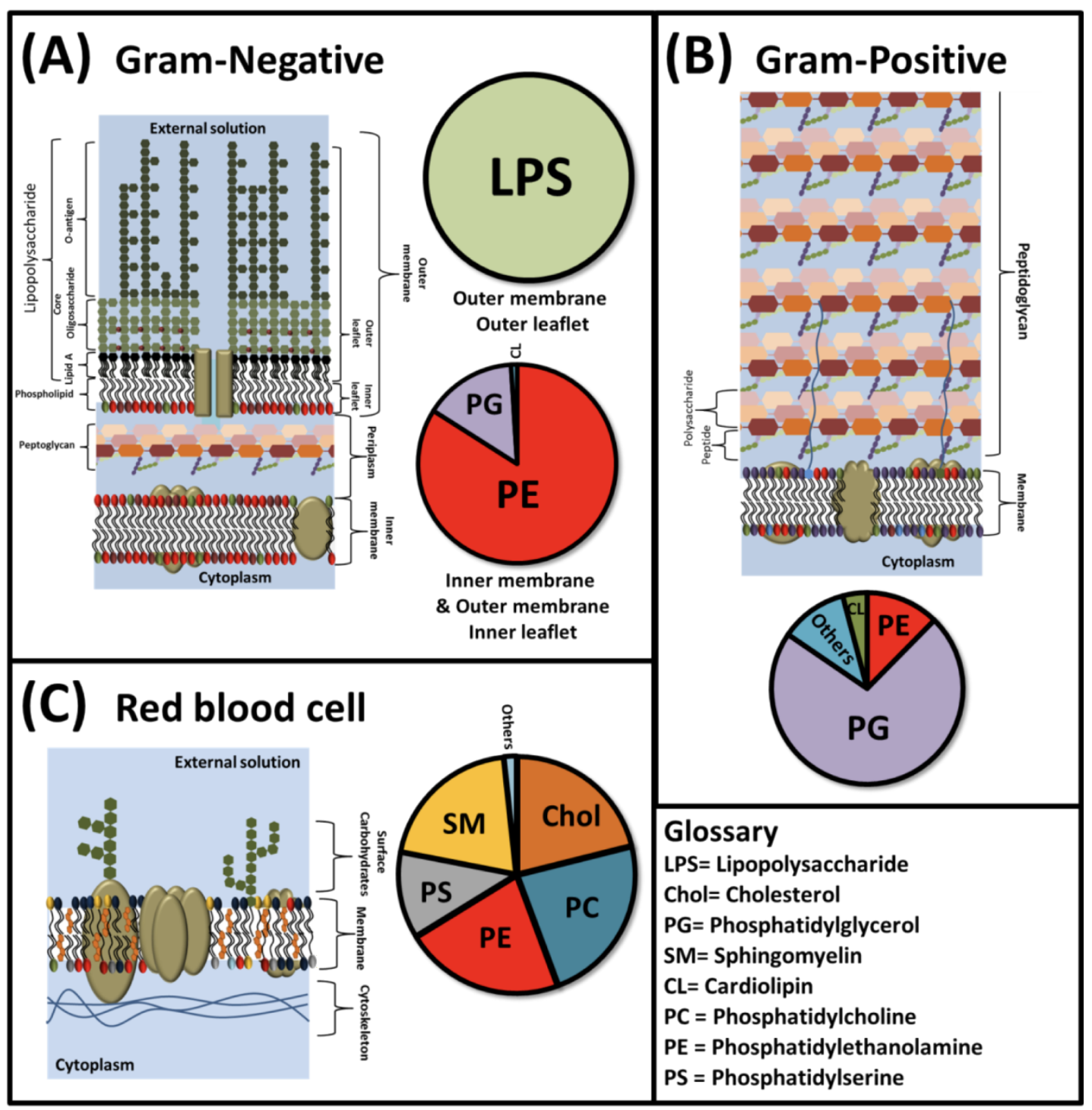

Figure 2. Schematic representations of cross sections of Gram negative (A), Gram positive (B) and erythrocyte (C) cell surfaces. Charts show the relative lipid composition of each membrane type. It should be noted that the values stated for Gram negative membranes are those reported for $E$. coli [32] and Gram positive are those reported for B.subtilis [33]. There is variation in the phospholipid composition across species but the values given are broadly representative. Further information on bacterial lipids can be found a review by Sohlenkamp and Geiger [34]. Red blood cell membrane lipid composition values are obtained from Vitanen et al. [35].

\section{Models of Cellular Membranes}


In this section we detail the current state of the art for planar membrane systems used as models of cellular membranes in biophysical interaction studies both at the air-liquid and solid-liquid interfaces. The relative advantages and limitations of all the models presented are described as well as details specific to a given sample type. All the models described are interfacial as surfaces are useful and convenient platforms both in the formation and study model cellular membranes.

\subsection{Lipid Monolayers at the Air-Liquid Interface}

Lipid monolayers at the air-liquid interface are typically considered to be the simplest model biomembrane systems as they contain just a single lipid leaflet of the bilayer. Lipids and sterols are poorly soluble in water, and readily form Langmuir monolayers (or films) at the air-liquid interface when deposited from solutions involving organic solvents $[36,37]$. The physicochemical behavior of a Langmuir monolayer can be studied via a surface pressure-area isotherm, which correlates the decrease in surface tension from the monolayer with its mean area per molecule (Figure 3). Practically, an isotherm is obtained by spreading on a Langmuir trough a given amount of lipid molecules so that the resulting monolayer is in the gas phase $(0 \mathrm{mN} / \mathrm{m})$, and then horizontal barriers slide to compress the monolayer by reducing the trough area while the surface pressure is monitored. The surface tension used to calculate the surface pressure is measured using a contact probe called a Wilhelmy plate [38], Figure 3A, while the trough is a shallow container made usually from PTFE (although the use of a hydrophilic material can reduce the volume) with barriers made either from PTFE or a hydrophilic material [39]. One or more linear or periodic cycles of the surface area can be performed and these are then known as compression/expansion isotherms [40].

Figure 3B gives the surface pressure-area isotherm for dipalmitoylphosphatidylcholine (DPPC). DPPC is probably the most studied phospholipid and constitutes the textbook example for the lipid monolayer phase behavior of a lipid monolayer at the air-liquid interface, passing from the gas phase through different phases such as liquid expanded (LE) and liquid condensed (LC), or a co-existence between the two, until a collapse pressure is reached. Measurements of the surface pressure of a phospholipid monolayer 
as a function of time can be performed with respect to its exposure to species introduced into the gaseous [41,42] or liquid phase. In the latter case, a range of molecular interactions have been studied including those of peptides [43], proteins [44], DNA [45], drugs [46] and nanoparticles [47]. Practically, injections of soluble species can be performed using a needle under the barriers [48], although this has the disadvantage that the concentration of species injected is not uniform and local high concentration gradients persist over long diffusion times. A flow trough was developed for efficient subphase exchange and to improve issues relating to inhomogeneity [49], but it relies on complete lack of dissolution of the monolayer. A further alternative is to spread the lipid directly on a subphase containing the soluble species [50].

There are several strengths of using lipid monolayers at the air-liquid interface as cellular membrane mimics such as the samples can be formed with complete and fully controllable lipid coverage, i.e. without defects, due to the lateral pressure and fluid nature of the membrane [51], and the monolayers are not subjected to unwanted charge interactions that a solid substrate can exert on a supported bilayer [52]. The primary limitation of work with monolayers is that there is only one membrane leaflet, so processes such as translocation cannot be studied [53]. Even so, the general approach has provided a wealth of useful information in a broad range of fields of study over the years, and as will be described below monolayers can be used practically in the preparation of supported bilayers. The approach remains a strong complement to others both at interfaces and in the bulk solution to the present day. 

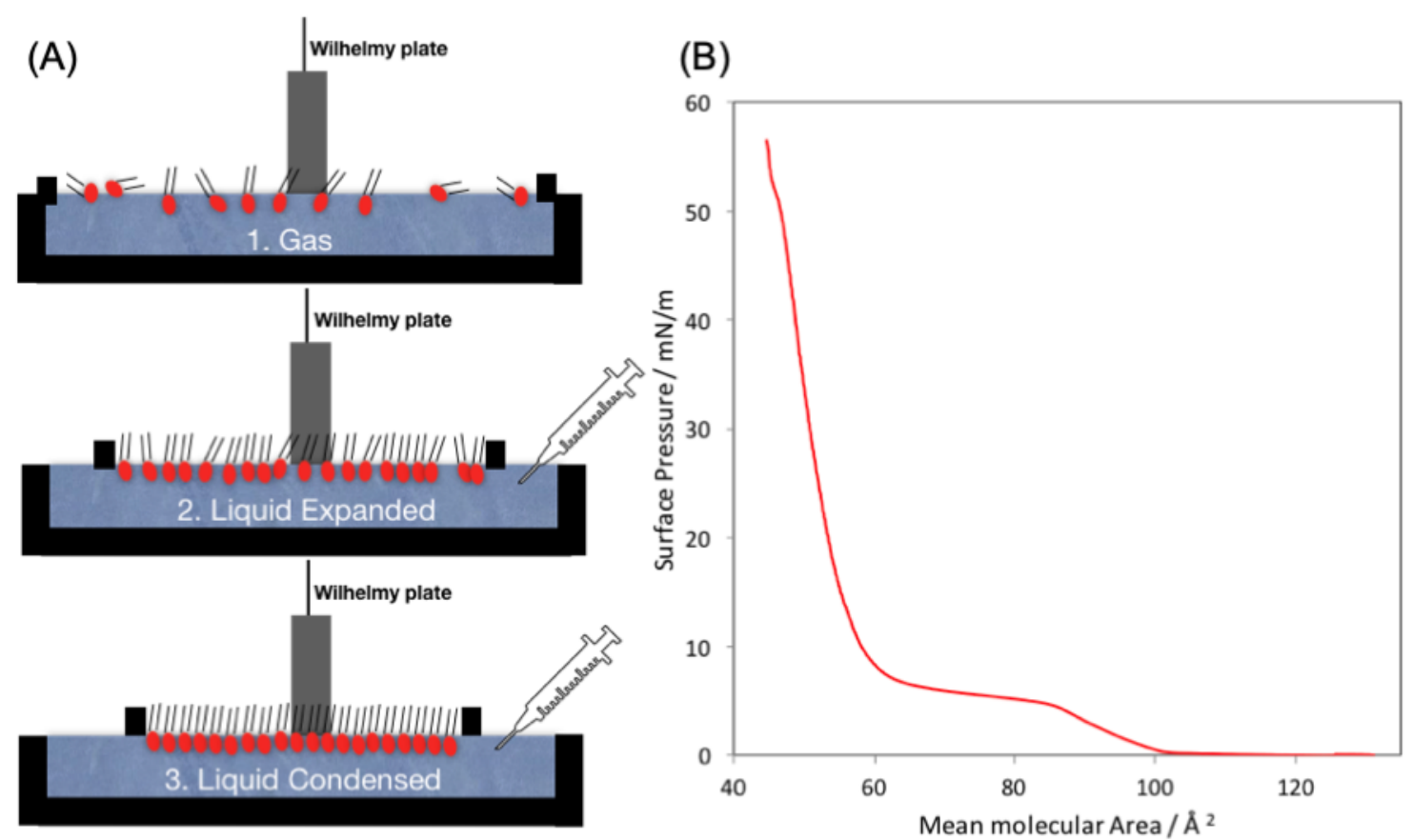

Figure 3. Langmuir trough (not to scale), sketch (A) and surface pressure-area isotherm for DPPC as a representative example (B). At $0 \mathrm{mN} / \mathrm{m}$ in surface pressure, lipids are arranged on lipid islands that do not cover completely the interface (gas phase). As the area is decreased, the lipids' acyl chains and head groups begin to orient and interact with each other, and the surface pressure 'lifts off' from its zero value (liquid expanded phase). Upon further compression, for lipids below their melting temperature, a plateau in the surface pressure could occur where the chains of lipid molecules condense in discrete domains (the liquid expanded-liquid condensed co-existence region). With further compression still, the surface pressure increases steeply as the monolayer becomes uniform yet again and the lipid chains are fully oriented and organized (liquid condensed phase). Lastly, at a maximal value of surface pressure, the film collapses into the aqueous subphase or forms multilayers. In the case of DPPC, the collapse does not occur until a surface pressure $>72$ $\mathrm{mN} / \mathrm{m}$ is reached, and the position of the surface pressure plateau in the isotherm is strongly dependent on temperature [54], ionic strength, $\mathrm{pH}$, and isotopic composition [55]. Biomolecules can be injected below the monolayer via the liquid phase using a syringe (A).

\subsection{Supported Lipid bilayers at the Solid-Liquid Interface}

Supported lipid bilayers (SLBs) represent the next stage of more realistic model biomembrane system at interfaces, as they contain two lipid leaflets. These samples are composed of a single lipid bilayer deposited onto a solid surface, which are often used to probe the interactions with molecules from solution [56]. The solid substrates used include 
glass, mica, silicon, sapphire, titanium oxide and gold (for recent reviews see articles by Lind and Cárdenas and Cho et al [56,57]). Even though their preparation and characterization are more involved than for monolayers, SLBs are widely used as models of cell membranes.

SLBs have been used to produce mimics of both eukaryotic and prokaryotic membranes $[56,57]$. For eukaryotes, $P C$ is typically used alone or in combination with other lipid types [58-60] such as PS [61-63], PG [14,15] and PI [64]. The lipid type and lipid species are tuned to ensure the fluidity and biological accuracy of the membranes [56]. Like Langmuir monolayers, the length and unsaturation level of the fatty acids in lipids have a role on the structure and properties of the resulting biomembranes $[58,60]$. The head group type in the lipids also influence the structure and properties of SLBs, when, for example, those with strong hydrogen bonding capacity (e.g. PE [65] and glycolipids [66]) are used since they increase the chain melting temperature $\left(T_{m}\right)$ of the lipids. A common lipid used in biomembranes is dimyristoylphosphatidylcholine (DMPC) with a $T_{m}$ close to $24{ }^{\circ} \mathrm{C}$ and saturated acyl chains. Thus, fluid membranes are only obtained above $24{ }^{\circ} \mathrm{C}$ [67]. If 1 palmitoyl, 2-oleoyl-sn-phosphatidylcholine (POPC), the most representative PC for mammalian cells, is used instead then fluid membranes exist even below room temperature. The use of cholesterol changes the fluidity of membranes due to the formation of liquid ordered phases. Cholesterol is typically used in combination of high and low $T_{m}$ lipids to mimic lipid rafts [68-71].

Fluid membranes of the main bacterial zwitterionic lipid type PE cannot be obtained with dimyristoylphosphatidylethanolamine (DMPE) at physiological temperatures $\left(\sim 37^{\circ} \mathrm{C}\right)$, as it has a $T_{m}$ close to $50^{\circ} \mathrm{C}$ [67]. Instead, 1-palmitoyl, 2-oleoyl-sn-phosphatidylethanolamine (POPE) should be used at $37^{\circ} \mathrm{C}$. As zwitterionic lipids, both PC and PE carry no net charge at neutral $\mathrm{pH}$. However, all natural cellular membranes have a certain content of anionic lipids (such as PI, PS, CL and PG). Bacterial membranes in particular contain high contents of the negative PG lipids (Figure 2), which are often used in simpler prokaryotic membrane models. More advanced and accurate bacterial models can be fabricated from bacterial polar lipid [72] or LPS [73,74] extracts. 
There are several methods to produce SLBs but the main two methods used are vesicle fusion or Langmuir-Blodgett deposition. The success of the vesicle fusion approach (Figure 4A-B) relies on several practical aspects that include the size of the vesicles, the underlying surface properties, the temperature and the ionic conditions [57]. Small unilamellar vesicles (SUVs) tend to fuse upon reaching the surface [56,58,72]. Glass, silicon, titanium oxide or mica are negatively charged and have favourable interactions with PC lipids, which lead to the adsorption of vesicles on the surface, their flattening and eventual collapse [75]. The experimental temperature is typically set above the $T_{m}$ of the lipids [58], although PC vesicles in the gel phase can also fuse and form SLBs of high coverage [76]. The ionic conditions of the bulk solution are extremely important to favor the deposition of vesicles containing charged lipids [57,64,72]. Two approaches have been applied that include the use of solutions with elevated ionic strength (typically 100$200 \mathrm{mM} \mathrm{NaCl}$ ) [64] or the use of divalent ions to bridge the vesicles to the surface (typically 2-5 $\mathrm{mM} \mathrm{CaCl}_{2}$ ) [72]. The use of salt solutions favors the formation of multilamellar vesicles, which can lead to excessive vesicle adsorption. $\mathrm{CaCl}_{2}$ has the disadvantage that vesicle aggregation due to $\mathrm{Ca}^{+2}$ bridging will occur over time, but this type of aggregation is reversible. Deposition on multilamellar stacks on the surface can be avoided by maintaining a constant flow of liquid through the measurement cell and diluting the solution as soon as good SLB surface coverage is obtained [77]. For example, SLB via vesicle fusion of polar bacterial extracts [72] and of POPE- 1-palmotoyl-2-oleoylsn-phosphatidylglycerol (POPG) lipids [78] can be achieved by using temperature above the $T_{m}$ of the lipids and 2-3 $\mathrm{mM} \mathrm{CaCl}_{2}$ under continuous flow. 


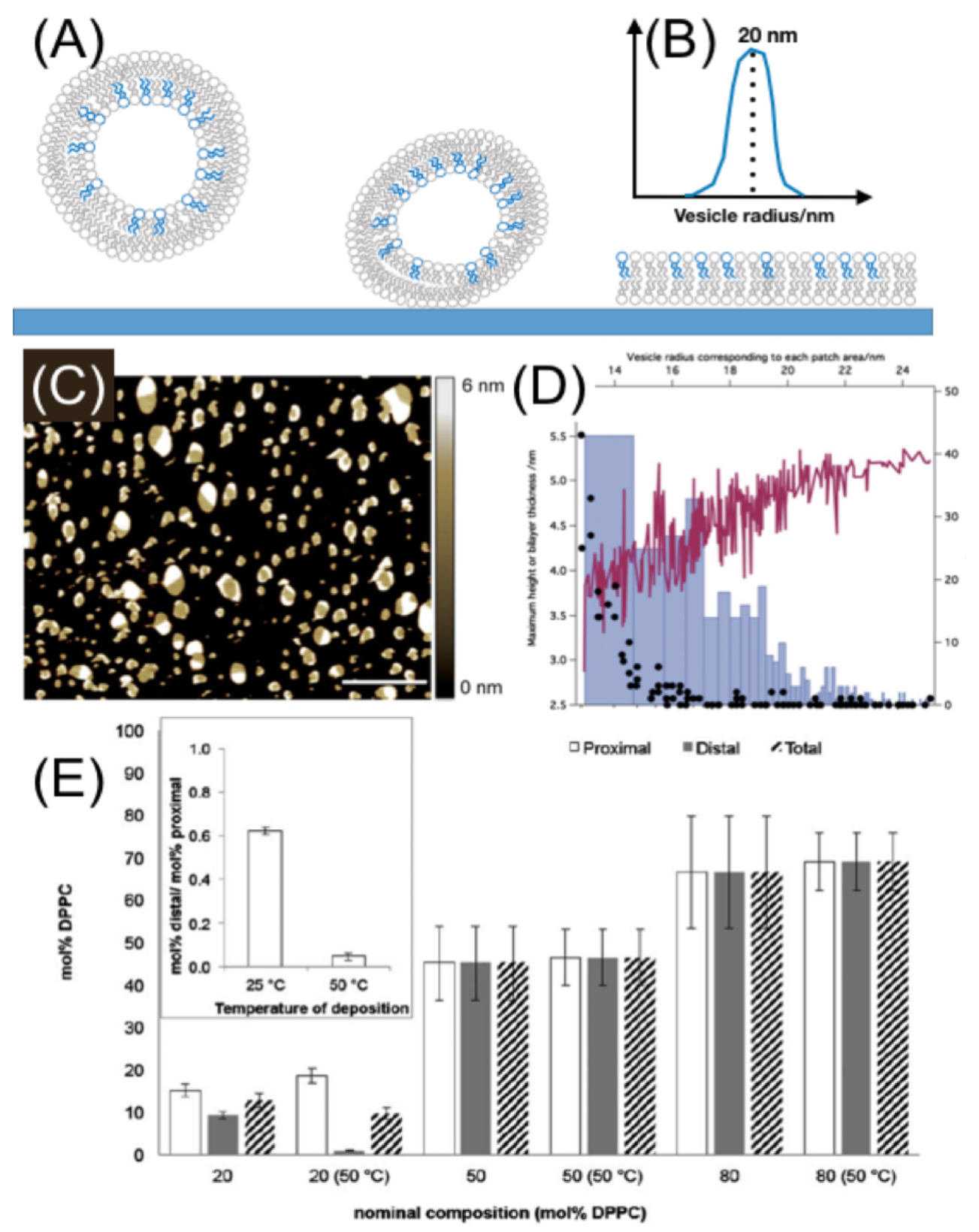

Figure 4. SLB formation via vesicle fusion includes vesicle adsorption, deformation and collapse (A). Small unilamellar vesicles (B) give good results, which due to spontaneous asymmetry in the smallest vesicles (C, D) can form SLBs displaying lipid asymmetry (E) when the mixture is close to the phase boundary between gel and fluid phases. Figure is adapted from ref. [58] permission from the Royal Society of Chemistry.

As previously mentioned, lipid asymmetry is an important characteristic of cellular membranes. Langmuir-Blodgett (LB) and Langmuir Schaefer (LS) deposition provides a 
controlled method for depositing both symmetric and asymmetric SLBs (Figure 5). This method uses the sequential deposition of lipid monolayers at the air-liquid interface (see section 3.1) onto a solid support material and therefore allows the content of each leaflet to be controlled at the point of deposition [79].

The advantage of SLBs is their robustness and reproducibility. Fabrication by vesicle fusion is a fast method and does not require specialised skills or apparatus. However, when using vesicles composed of two or more lipid components, symmetric composition between the two bilayer leaflets is often achieved. Some exceptions occur where spontaneous asymmetry and deviation from the nominal composition of the vesicles used to form the SLB occur $[58,60,63,68]$. For example, spontaneous asymmetry is found in lipid mixtures having components with high and low $T_{m}$ when working close to the phase boundary between fluid and gel phases $[58,60,68]$. In this case the asymmetry results from selective adsorption of the smallest vesicles within the total vesicle population [58] which tend to have an asymmetric lipid composition due to their high curvature $[80,81]$ (Figure 4C-E).

Membrane leaflet asymmetry that truly mimics that found in native cellular membranes $[20,82]$ can only be achieved by LB-LS method since each leaflet is deposited separately. This method has been used to deposit asymmetric models of the Gram negative bacterial outer membrane $[73,83]$ where phospholipids are deposited as the proximal (inner) leaflet and bacterial LPS are deposited as the distal (outer) leaflet as well as model fungal membranes where a different phospholipid composition is deposited in each leaflet [82]. Deuterium isotope labelling of individual leaflets within membrane deposited by LB-LS allows for studies on the relative changes in membrane organisation under differing conditions using isotope sensitive techniques such as NR [74,84] and ATR-FTIR [80], as will be introduced in section 5.2 . 


\section{Langmuir Blodgett}

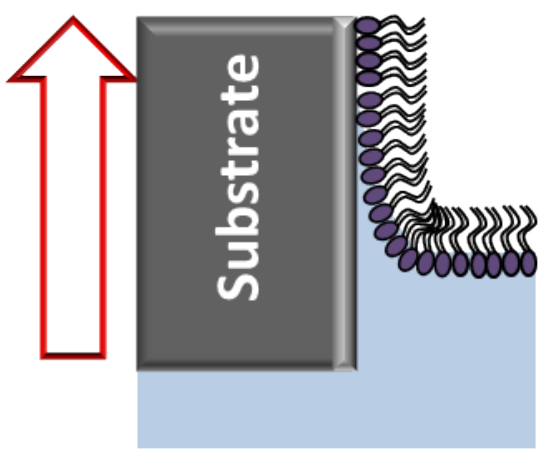

Langmuir Schaeffer

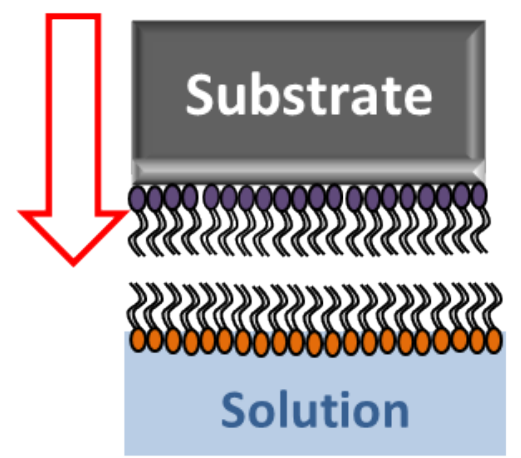

SLB

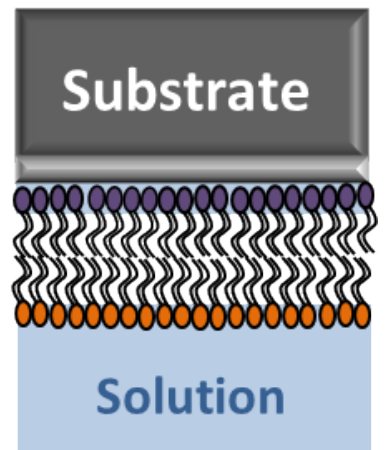

Figure 5. Langmuir-Blodgett/Langmuir-Schaefer deposition of sequential air-liquid interfacial monolayers to produce an asymmetric SLB. The initial step in the membrane fabrication is to deposit the proximal (inner) leaflet of the bilayer by Langmuir-Blodgett deposition. The surface pressure is taken to $<35 \mathrm{mN} \mathrm{m}^{-1}$, and the lipids are deposited onto the solid interface from the monolayer "head group first": the surface becomes hydrophobic due to the acyl chains facing the external environment. Next, Langmuir-Schaefer deposition is used to deposit the distal (outer) leaflet of the bilayer. A second lipid monolayer is spread onto the water surface and compressed to a high surface pressure. The LB coated substrate surface is then held parallel above a lipid monolayer coated air-liquid interface. The substrate is lowered through the interface. As the substrate meets the air-liquid interface, the acyl chains of the LB deposited monolayer and the acyl chains of the monolayer at the air-liquid interface come into contact to form a complete SLB. This SLB is instantly immersed in solution as the substrate is lowered through the water interface and, usually, assembled into a solid-liquid flow cell under water for further use.

The disadvantage of SLBs is that they are in close contact with the solid surface and therefore do not have significant reservoirs of solution on both sides of the membrane as would be found in vivo. The close proximity of the substrate to SLBs affects the diffusion of lipids and proteins as compared to free-standing bilayers [85]. This limitation is due to the fact that the substrate exerts greater influence on the behavior of the proximal leaflet than the distal leaflet of the SLB, due to its closer proximity to the surface $[86,87]$. The roughness of the substrate and the complementarity between the surface and lipid charge will determine the magnitude of the surface influence (for a review see ref. [88]). Substrates such as quartz, mica and silicon (with its thin $\mathrm{SiO}_{2}$ coating) are anionic which can make the deposition of model membranes with high amounts of negatively charged 
lipids in the proximal leaflet challenging due to electrostatic repulsion [72]. Additionally, these charged surfaces may exert attractive or repulsive forces against molecules in solution through defects present within the SLB. Lastly, membranes naturally fluctuate [89] and these fluctuations are suppressed in SLBs due to the close proximity of the bilayer to the substrate surface [90]. Defects, however, can actually be advantageous to pick up more easily small morphological changes occurring on an otherwise homogeneous surfaces using atomic force microscopy [91] (see section 5.2).

\subsection{Advanced Planar Membrane Systems}

For the reasons stated above, more advanced planar model membranes have been developed where the substrate interactions are minimized with an aim to produce more accurate bio-mimetic systems with significant solution reservoirs on both sides of the bilayer. These planar membrane models can be broadly categorised into three types: cushioned, tethered and floating membranes.

In cushioned bilayers, a polymer layer between the planar bilayer and the solid-liquid interface acts as a lubricating spacer to prevent bilayer surface interactions [92] (Figure $6 A)$. Requirements of the polymer support are that it must be hydrophilic and have no interactions with the membrane constituents [93]. Reconstituted cellulose is commonly cushion material [92] although polyacrylamide, polylactic acid, agarose, PEGylated lipids and polyelectrolytes are also used [94,95]. The thickness of the support cushions can range from a few nanometers to hundreds of microns. The cushions are hydrophilic and act as a solution reservoir. Compared to SLBs, polymer supported bilayers show better lateral mobility of the membrane components $[95,96]$.

Tethered lipid bilayers are a class of membrane model where a single bilayer is held close to solid support interface through surface attached molecules in the proximal leaflet of the bilayer (Figure 6B). In its simplest form these can be hybrid bilayers with a surface grafted inner leaflets $[97,98]$. More advanced models use a substrate grafted hydrophilic polymer which is terminated by a lipophilic anchor molecule [97-99]. The lipid anchor molecules can either form a complete inner leaflet [97] or, preferentially, form a minor component of it [100]. To achieve a lower coverage of anchor molecules across the solid support, the 
tether molecules are interspaced with another covalently grafted self-assembled monolayer species. A low tether coverage allows for bulk solution between the surface and bilayer [98]. Tethered SLBs have found applications in membrane interaction studies $[97,98]$. The ease of self-assembling the membranes onto technologically relevant surfaces such as gold $[97,101]$ have made these a popular membrane mimetic system that is amenable to characterisation by a wide variety of analytical techniques.

Floating SLBs offer, perhaps, the most realistic planar mimetics of a biological membrane (Figure 6C). In this sample type a membrane is deposited above an SLB $[102,103]$ or a grafted lipid self-assembled monolayer [104,105]. A combination of repulsive entropic hydration, electrostatic and fluctuational (Helfrich) forces and attractive Van der Waals forces [103] cause the bilayers to float 2-3 nm away from the surface deposited material. As these bilayers have solution reservoirs on both sides and no anchoring or support materials they freely fluctuate [106] in the same way as is observed with native membranes. Due to short range repulsive forces, samples can only be prepared by sequential LB and LS depositions. These methods preclude the inclusion of integral membrane proteins in the model and can present difficulties with the deposition of airsensitive lipid species. However, recently a methodology was developed which allows for floating bilayers to be self-assembled onto surfaces by vesicle rupture, which opens up new possibilities for studies with this sample type [107].

Cushioned, tethered and floating bilayers have a distinct advantage SLBs due to the reduction of surface induced effects. Additionally all three advanced membrane types allow the deposition of anionic lipid in both leaflets, which is difficult with most common surfaces for SLB deposition (mica, quartz and silicon). Tethered and cushioned bilayers offer the ability to increase the accuracy of the membrane mimetics through the inclusion of transmembrane proteins, which gives rise to a myriad of new opportunities in the study of membrane biochemistry. 


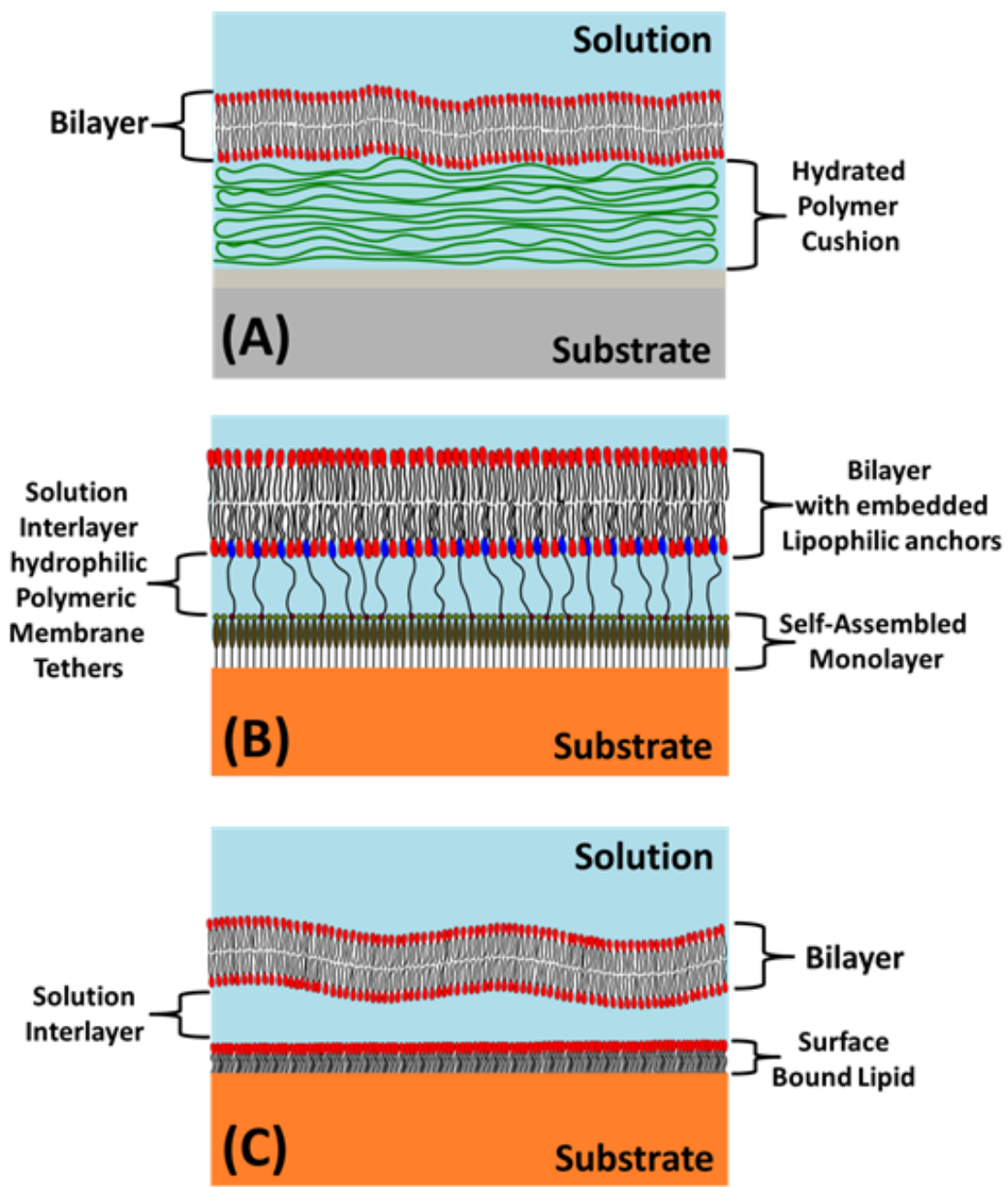

Figure 6. Schematic representations of cushioned (A), tethered (B) and floating (C) bilayers. Tethered and floating bilayers are, in general, deposited from vesicle suspensions while floating bilayers are deposited by LB-LS.

The disadvantage of these advanced biomembrane models is the relative complexity of producing the samples. SLBs of very high quality can be fabricated with ease by vesicle rupture onto glass, mica or silicon. While cushioned, tethered and floating bilayers each have several extra steps in the deposition making the sample fabrication difficult for the novice. Another alternative for a close-to-native condition in SLBs is the use of ordered porous solid surfaces as substrates for membranes of lateral dimensions higher than those of the pore periodicity [108]. Thus, regions of the membranes covering the holes will be non-supported. 


\section{Surface-sensitive Techniques to study Molecular Interactions at Model Biomembranes}

In this section, we will detail the use of a range of various modern experimental methods in the study of molecular interactions with biomembranes. The use of each technique is demonstrated through the consistent example of the interactions of antimicrobial peptide (AMP) Melittin (MLT), a membrane-disrupting peptide produced in the venom of European honey bees (Apis mellifera), with planar models of Gram positive bacterial membranes (composed of DMPC and DMPG).

$\mathrm{MLT}$ is a 26 amino acid long peptide that carries +6 charge at physiological $\mathrm{pH}$. This peptide is very potent against Gram positive bacteria (the minimum inhibitory concentration, MIC, is $2-4 \mu \mathrm{g} / \mathrm{mL}$ [109]), but it has a high hemolytic activity at $128 \mu \mathrm{g} / \mathrm{mL}$ and lower activity against Gram negative bacteria (MIC = 16-64 $\mu \mathrm{g} / \mathrm{mL}$ [109]). MLT adopts an alpha-helical structure in solution and is proposed to create channels in the membrane leading to its solubilisation: Its interaction with model biomembranes (both monolayers and bilayers) is well documented in the literature [110-118]. While the peptide is wellstudied in the literature, studies vary widely in the experimental conditions employed. A comparison of the data from each technique recorded under similar experimental conditions is described and a precision understanding of the MLT/membrane interaction is resolved as a result, providing the reader with an example of how to combine techniques to elucidate complex interaction phenomena.

All of the measurements were performed in $50 \mathrm{mM}$ Tris buffer $(\mathrm{pH} \mathrm{7.4)}$ at room temperature. The charged PG to zwitterionic PC lipid ratio was kept at a molar ratio relevant for Gram negative membranes (Figure 2) but the specific lipid varied among the techniques: POPC and POPG were used to better resemble natural lipid chains for all techniques except those requiring deuterated lipids, and a three component lipid mixture containing DPPC was used in AFM to allow the formation of lipid domains and defects for easier visualisation of the bilayer and the initial moments of MLT interaction with the SLB. We must stress that the methods hereby discussed complement the information accessible through computer simulations that give detailed information on the 
oligomerization state of AMPs at the membrane, the degree of penetration on the lipid membrane as well as the specific groups interacting with each other, see for example refs $[45,59,119,120]$.

\subsection{Changes in Interfacial Mass from Quartz Crystal Microbalance with Dissipation Monitoring and Ellipsometry}

Quartz crystal microbalance with dissipation monitoring (QCM-D) is a sensitive method for mass determination of materials adsorbed on a piezoelectric single crystal quartz sensor [121]. Resonance is achieved when the standing wave produced by the alternating expansion and contraction of the piezoelectric crystal is an odd integer of the thickness of the quartz sensor, which means that different harmonics (i.e. overtones, $n$ ) can be measured. The resonance frequency, $f$, gives information on the adsorbed mass on the quartz sensor. For rigid and homogeneously distributed adsorbed films, the mass of the film is inversely proportional to the resonance frequency of the quartz crystal via the so-called Sauerbrey equation [122]. There are several reviews on QCM-D, e.g. the recent one Alassi et al [123].

The technique can also be used to measure the dissipation, $D$, of an interfacial film, which is related to the energy lost (dissipated) in relation to the stored energy. This parameter can be quantified by measuring the decay time of the oscillation of the quartz sensor when the alternating potential is turned off, which is related to the energy dissipation of the quartz resonator and greatly depends on the viscoelastic properties of the film on the sensor surface [124]. The technique has thus the additional capacity to provide information on the viscoelastic properties of the adsorbed material [124]. The combination of experimental data on $f$ and $D$ from several overtones allows the extraction of useful parameters through data modeling, such as the adsorbed mass, film thickness, or viscoelastic properties. For viscous interfacial films, the Sauerbrey equation [122] does not apply, and it is necessary to use more sophisticated modeling approaches such as the Voigt model [125].

QCM-D measurements indicate three characteristic features associated with completion of SLBs via vesicle fusion: (1) the initial regime where large increases in mass ( $f$ 
decrease) and in $D$ due to adsorption of intact, water filled vesicles are observed, followed by (2) a decrease of both mass ( $f$ increase) and dissipation due to rupture of vesicles and associated release of trapped water, and lastly (3) fusion into a rigid lipid bilayer where the frequency and dissipation data reach stable values (Figure 7C). The effects on $f$ and $D$ over time upon addition of $5 \mu \mathrm{M}$ MLT to an SLB made of POPC-POPG is shown in Figure 7D; initially, the presence of MLT leads to a small increase in $f$, followed by an abrupt decrease-increase change, which finally stabilises at $f$ values slightly lower than those corresponding to the intact SLB. A mirroring effect is seen for $D$. However, in the final stable region, a significant spreading of the dissipation data from the different overtones is observed. Taken together, the $f$ and $D$ data in Figure $7 C$ and $D$ suggest that MLT initially adsorbs to the SLB, which leads to a re-arrangement of the surface structure of the SLB and potentially desorption of lipids. Ultimately, the MLT interacts with the SLB leaving an adsorbed lipid layer that is probably more heterogeneous and softer than the original SLB.
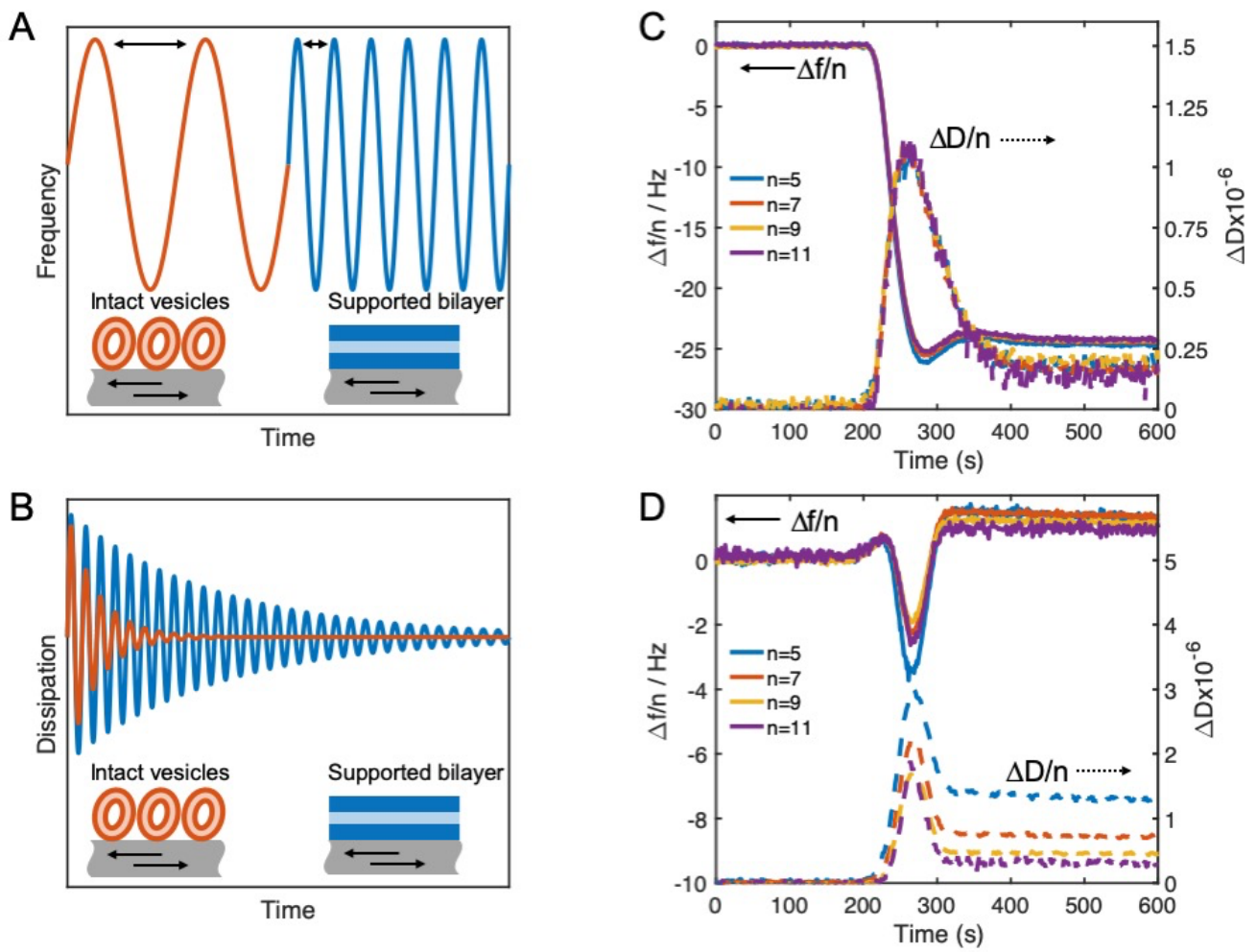

Figure 7. Schematics showing that signals of the frequency (A) and dissipation (B) from QCM-D depend on the nature of the interfacial film including the adsorbed amount and viscoelastic properties. In the example, data are shown during formation of an SLB made from 8:2 POPC:POPG 
vesicles by molar ratio in $2 \mathrm{mM} \mathrm{CaCl}$ by passing the solution through the measurement cell from $\mathbf{t}$ $=200 \mathrm{~s}(\mathrm{C})$, which was followed by rinsing through the measurement cell with $50 \mathrm{mM}$ Tris buffer (pH 7.4), and lastly the SLB was exposed to $5 \mu \mathrm{M} M L T$ in $50 \mathrm{mM}$ Tris buffer (pH 7.4) from $t=200 \mathrm{~s}$ (D).

Ellipsometry is an optical technique based on the measurement of changes in the polarization of light reflected at a planar interface where it is possible to obtain the adsorbed mass of an interfacial film and in cases its thickness [126]. The polarization of the reflected light is modulated by the optical properties of the bulk media and the optical properties of any film at the interface as well as instrumental parameters such as the wavelength and angle of incidence. The typical PCSA (Polarizer-Compensator-SurfaceAnalyzer) setting for a null ellipsometer is depicted in Figure 8A $[126,127]$. Here, the polarizer produces linearly polarized light for which the plane of polarization can be adjusted. The linearly polarized light goes through a compensator that induces a phase shift between the two component light waves of $\pi / 2$ radians (or one quarter of the wavelength) so that elliptically polarized light is obtained. The ellipticity of the incoming light is regulated by the polarizer in such a way that the light after reflection at the surface is linearly polarized. The analyzer is then adjusted so that the light intensity to the detector is minimized. The polarizer and analyzer positions at minimal light intensity to the detector gives the ellipsometric angles, $\Delta$ and $\Psi$. Here $\Delta$ gives the relative phase shift of the perpendicular components of elliptically polarized light while $\Psi$ gives the corresponding amplitude differences. A null ellipsometer typically has a laser source, but there are also those with a bulb source and spectroscopic instruments. In addition to null ellipsometers, there are phase modulated ellipsometers where the light polarization is oscillated on the $\mathrm{MHz}$ time scale through the use of a birefringence modulator, and with no rotating optics this method can lead to faster data acquisition.

For interfacial films at the solid-liquid interface where the solid medium absorbs light (e.g. silicon wafers), modeling of $\Delta$ and $\Psi$ data can result in resolution of both the refractive index and thickness of the film. From these quantities, the surface excess can be calculated through knowledge of the mean refractive index increment $(\mathrm{dn} / \mathrm{dc})$ of the film [128] or the molar refractivity and partial specific volume of the adsorbed molecules [129]. For very thin films there is a co-variation of the refractive index and thickness, which is 
compensated in the mass calculation. This means that the mass can be determined with higher accuracy than the refractive index and the thickness individually [129]. With time, the concept of thorough characterisation of the substrate surface, i.e. the thickness of the oxide layer for a silicon substrate, was introduced based on measurements in different ambient media [130]. This method results in a reliable determination of thickness, refractive index and adsorbed mass [130,131]. Sensitivity of both parameters to the presence of an interfacial film can be enhanced if the silicon wafer has a thermally grown oxide layer since a larger change in both ellipsometric angles are obtained in this way. Ellipsometry, however, does not readily distinguish different components of a mixed system. The most commonly applied model for a thin layer on a flat solid surface is shown in Figure 8B. Here all interfaces are assumed to be planar (effects of substrate roughness are incorporated into the bare surface characterization [132]), and the film is uniform with a refractive index of $n_{1}$ and thickness of $d_{1}$ in between two media with different refractive index $\mathrm{n}_{0}$ and $\mathrm{n}_{2}$. 

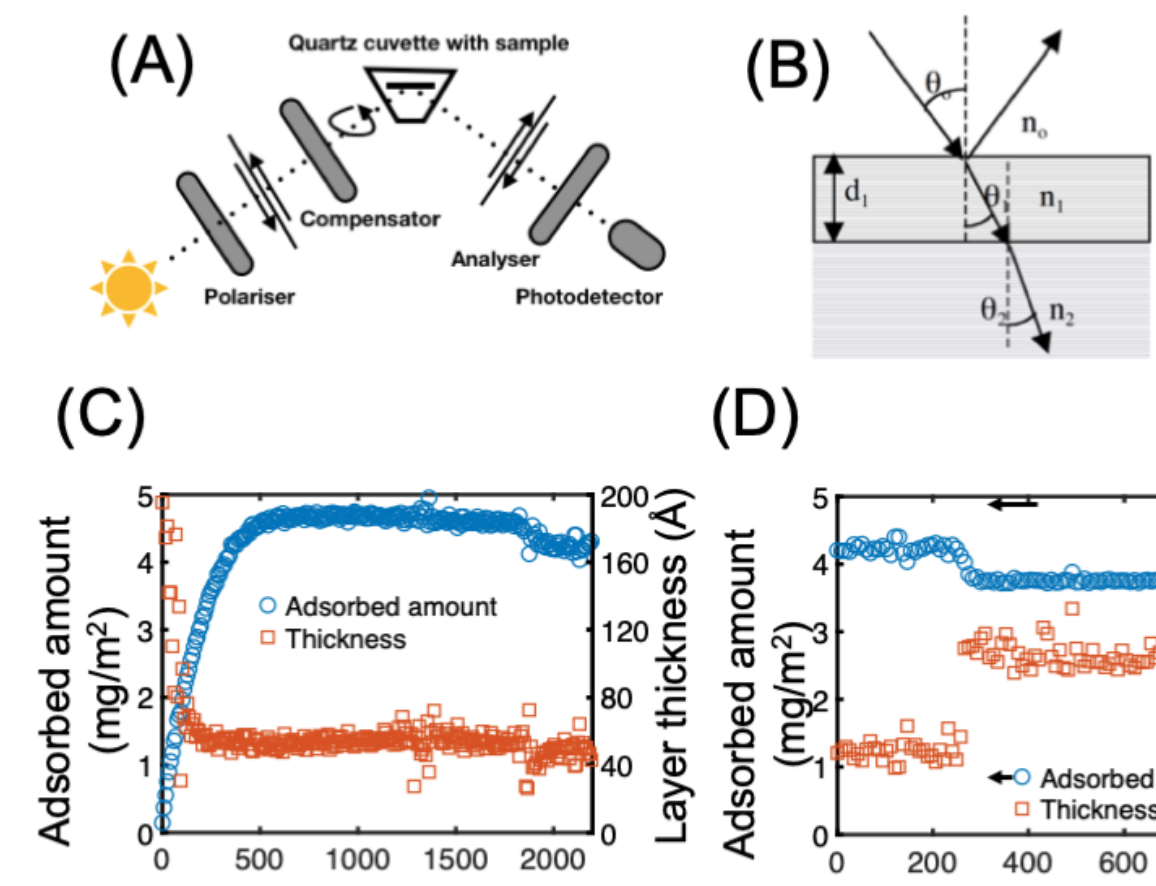

(D)
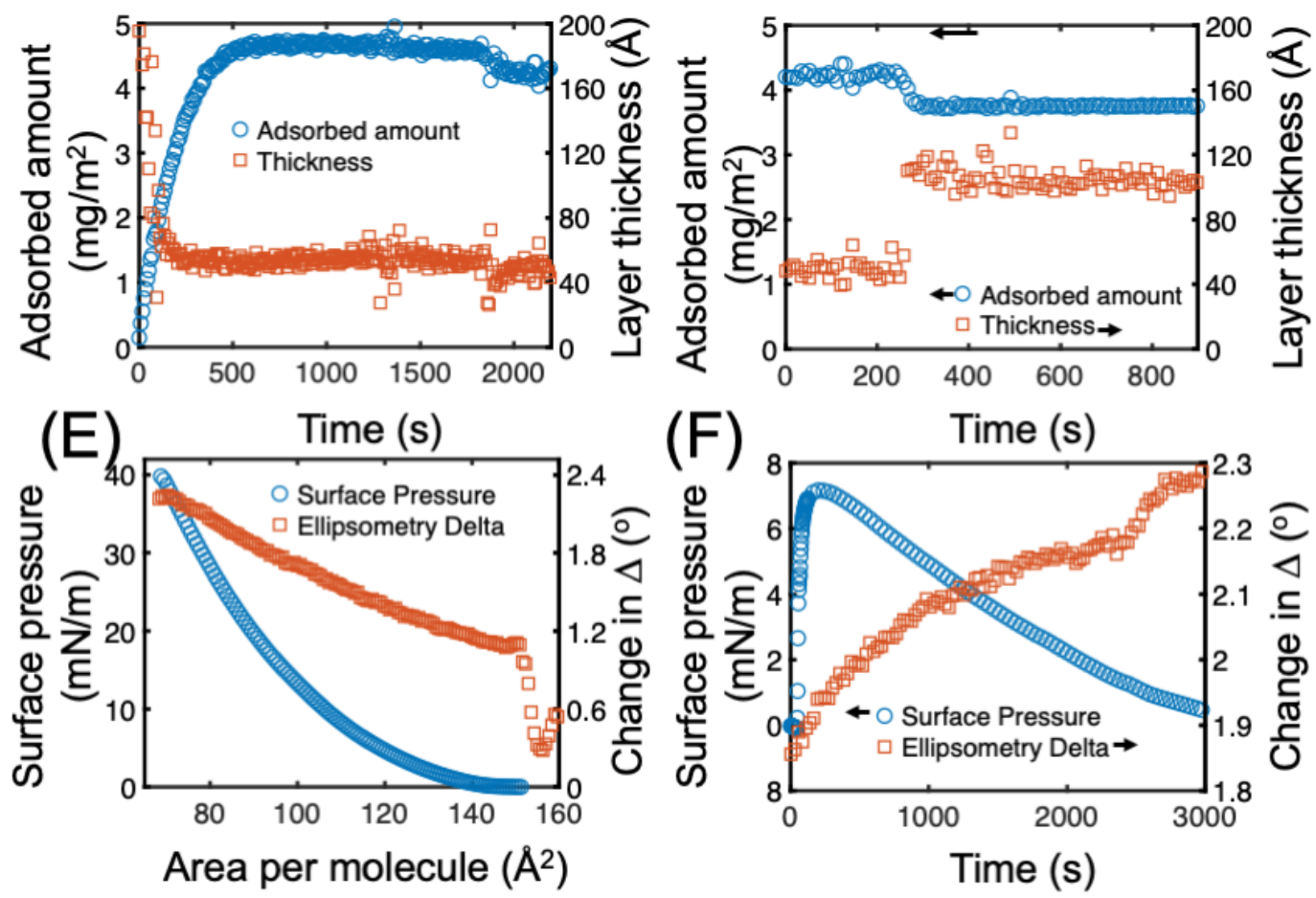

Figure 8. Schematic of the PSCA setup of a null-ellipsometer (A), and a typical single-layer model used for interpreting ellipsometry data (B). In the first example, data of the adsorbed amount (blue) and layer thickness (orange) are shown during formation of an SLB made from 8:2 POPC:POPG vesicles by molar ratio in $2 \mathrm{mM} \mathrm{CaCl}$ followed by rinsing through the measurement cell with $50 \mathrm{mM}$ Tris buffer $(\mathrm{pH}$ 7.4) from $t=1800 \mathrm{~s}(\mathrm{C})$, and upon its exposure to $5 \mu \mathrm{M} M L T$ in $50 \mathrm{mM}$ Tris buffer (pH 7.4) passed through the measurement cell from $t=200 \mathrm{~s}$ (D). In the second example, an 8:2 POPC:POPG by molar ratio monolayer on $50 \mathrm{mM}$ Tris buffer exhibits a steady increase in the ellipsometry signal (orange) over the whole recorded surface pressure-area isotherm (blue) indicating that the monolayer remains in the LE phase (E), and data of the surface pressure and change in $\Delta$ from ellipsometry upon exposure of a final concentration of $5 \mu \mathrm{M}$ MLT injected in aliquots into the solution under the monolayer with a starting surface pressure of $30 \mathrm{mN} / \mathrm{m}$ while keeping the trough area constant $(F)$. 
Ellipsometry has been extensively applied to follow SLB formation both via vesicle fusion [133] and the so-called mixed micelle method [134]. Figure $8 \mathrm{C}$ shows the change in mass and thickness over time upon addition of POPC:POPG vesicles to a 8:2 molar ratio in 2 $\mathrm{mM} \mathrm{CaCl}$. In contrast to $\mathrm{QCM}-\mathrm{D}$, the adsorbed amount increases steadily upon reaching $4.8 \mathrm{mg} / \mathrm{m}^{2}$ and the thickness remains rather constant at $5 \mathrm{~nm}$. This indicates that fusion occurs readily upon addition of the vesicles and that ellipsometry is only sensitive to the dry adsorbed mass (i.e. the lipids in the adsorbed layer). Ellipsometry has also been applied to study the effect of antimicrobial compounds to SLBs [135-137] as a function of the properties of the lipid membrane. In Figure 8D we show the effect of adding $5 \mu \mathrm{M}$ MLT to a preformed SLB. Immediately upon injection, there is a small increase in adsorbed amount followed by a decrease to slightly lower values than for the bare SLB. Additionally, the layer thickness seems to increase to twice the value of the SLB. This would imply that MLT initially adsorbs and readily leads to a layer rearrangement that is less dense than the original SLB (lower adsorbed mass and thicker layer).

For interfacial films at the air-liquid interface (where both bulk media are transparent), the parameter $\Psi$ is rather insensitive to the presence of the film. Thus, data treatment at the thin film limit is often carried out only on the change in $\Delta[138,139]$, i.e. the value of $\Delta$ for the sample minus that of pure solvent (with some notable exceptions, e.g. ref. [140]). This limits the type of information that can be extracted from ellipsometric measurements at the air-water interface to the adsorbed amount (through knowledge of the optical properties of an interfacial film). The film thickness can though be estimated by making assumptions about the film refractive index and density [141]. A further caveat of the approach is that the technique is highly sensitive to film anisotropy, hence simple model assumptions (involving an isotropic film) tend to break down for lipid systems according to their phase $[142,143]$. Indeed, the same is true at the solid-liquid interface but the problem is usually not considered in the data analysis. Nevertheless, with careful interpretation, the technique can provide important information about the extent and rate of interactions of molecules from solution with lipid monolayers. Furthermore, as the reflected beam is local $\left(\sim 1 \mathrm{~mm}^{2}\right)$ and data acquisition rapid (second time scale for a null ellipsometer; millisecond time scale for a phase modulated ellipsometer), fluctuations in 
the signal can reveal lateral inhomogeneity in the interfacial film [144,145], where other techniques such as specular neutron reflectometry are space-averaged over the centimeter length scale.

Figure $8 \mathrm{E}$ shows the surface pressure isotherm and the corresponding ellipsometric change in $\triangle$ for a monolayer of POPC:POPG 8:2 molar ratio in $50 \mathrm{mM}$ Tris buffer at room temperature. As the monolayer is compressed, the surface pressure isotherm presents no kinks, which indicates that there is no abrupt phase transition of the lipid molecules into the condensed phase (in contrast to the case of DPPC [146]; see Figure 3B). This observation is consistent with both of the lipids existing in the fluid phase at room temperature The ellipsometry data show a steady increase in ?? as the monolayer is compressed without any sharp change in gradient that could indicate the emergence of anisotropy in the monolayer.

Upon injection of $5 \mu \mathrm{M}$ MLT into the solution underneath the lipid monolayer prepared at a starting surface pressure of $30 \mathrm{mN} / \mathrm{m}$, while keeping the corresponding Langmuir trough area constant, there is a rapid and sharp increase in surface pressure accompanied by a steady increase in $\Delta$ over time. Thus, there is a clear interaction between the peptide and the lipid monolayer which increases the surface packing density. However, after 5 min there is a turnpoint where the surface pressure starts to fall while the ellipsometric $\Delta$ keeps on increasing over time. While these two techniques are not sensitive to the relative amounts of individual components at the interface, these observations hint that there is an interfacial rearrangement with a loss of lipid from the monolayer accompanying the peptide interaction. After $1 \mathrm{~h}$ of equilibration, there is a sharp increase in $\Delta$. This feature indicates that the film has become anisotropic due to the formation of lateral domains that have different compositions or ordering. The application of other techniques such as BAM or NR may allow elaboration of these possibilities, although this has not been carried out in the present work.

\subsection{Imaging Interfacial Morphologies using Atomic Force Microscopy and Brewster Angle Microscopy}


Atomic Force Microscopy (AFM) is an essential tool for the study of cellular and model biological membranes. Some unique properties are its ability to visualize these systems in native environments, without the need for fixation, staining, or labeling, and with a superior signal-to-noise ratio than any optical or electron microscopes. AFM imaging is performed by raster scanning the surface of a sample with a sharp tip attached at the free end of a soft micrometer-sized cantilever (Figure 9A). Imaging with $<1 \mathrm{~nm}$ vertical resolution is the AFM standard (lateral resolution, which is commonly limited by the size of the tip, lies within a range of ca. 2-50 nm). There are several AFM operation modes that allow 3D images of the surfaces to be obtained: in Contact Mode AFM [147] scanning is performed while keeping the deflection of the cantilever and, therefore, the applied load constant. This mode is associated with high shear forces, which can be minimized by operating the AFM in the so-called dynamic operation modes [148], which include the popular Amplitude Modulation AFM. In this mode, the cantilever is mechanically excited at a frequency close to its free vibrational resonance. Upon the presence of an interaction between tip and sample, the resonance of the cantilever shifts and, subsequently, so does the oscillation amplitude, which is the feedback signal that is kept constant during sample visualization. For a recent overview on AFM operation modes see ref. [149]. AFM is not only an imaging technique but can be used to probe and even map tip-sample interactions via, for example, force-distance curves [150]; for excellent reviews see refs [151-154]. If the cantilever is considered as a spring with an experimentally measured spring constant $[155,156]$, its deflection is directly related to the force exerted by the sample on the tip. Force-distance curves provide a measurement of both chemical and mechanical properties of SLBs: the membrane stiffness can be determined by monitoring how the cantilever is deflected when pushing it against the membrane [157]. Moreover, the tip will break into the bilayer at enough applied load [158] and the breakthrough force for this event has been extensively used to characterize supported model membranes as it represents a direct measurement of their mechanical stability at the nanoscale [159-161]. Force-distance curves and images can be combined by, e.g. the acquisition of a force curve at every point of the $2 \mathrm{D}$ scan, providing maps of properties of the samples, e.g. stiffness [162], breakthrough force [163] and adhesiveness [164]. 
Dynamic operation modes are often used to image biomembranes due to mechanical deformation that occurs when lipid membranes are scanned in the contact mode [165], which results in an underestimation of the membrane thickness [159]. AFM has sufficient resolution to resolve even individual head groups in SLBs [166] as well as lateral inhomogeneities within the membranes: these include surface defects (holes) [167], typically used for quantifying bilayer thickness, and phase separation (domain formation), e.g. in the LC-LE co-existence region for stearic acid [168] or mixtures of disterylphosphatidylethanolamine (DSPE) and dioleoylphosphatidylethanolamine (DOPE) [158]. The first AFM studies of model lipid membranes investigated samples prepared by LB deposition. However, the vesicle fusion technique soon became more popular $[169,170]$ boosting the use of AFM to study the phase behavior of phospholipid mixtures. Since then, the imaging capabilities of AFM have expanded its use allowing, for example, the visualization of modulated phases of transmembrane peptides incorporated in lipid bilayers [171,172] and upon continuous flow [91] (Figure 9B). Moreover, timelapse AFM, has seen some development thanks to High Speed implementations. 

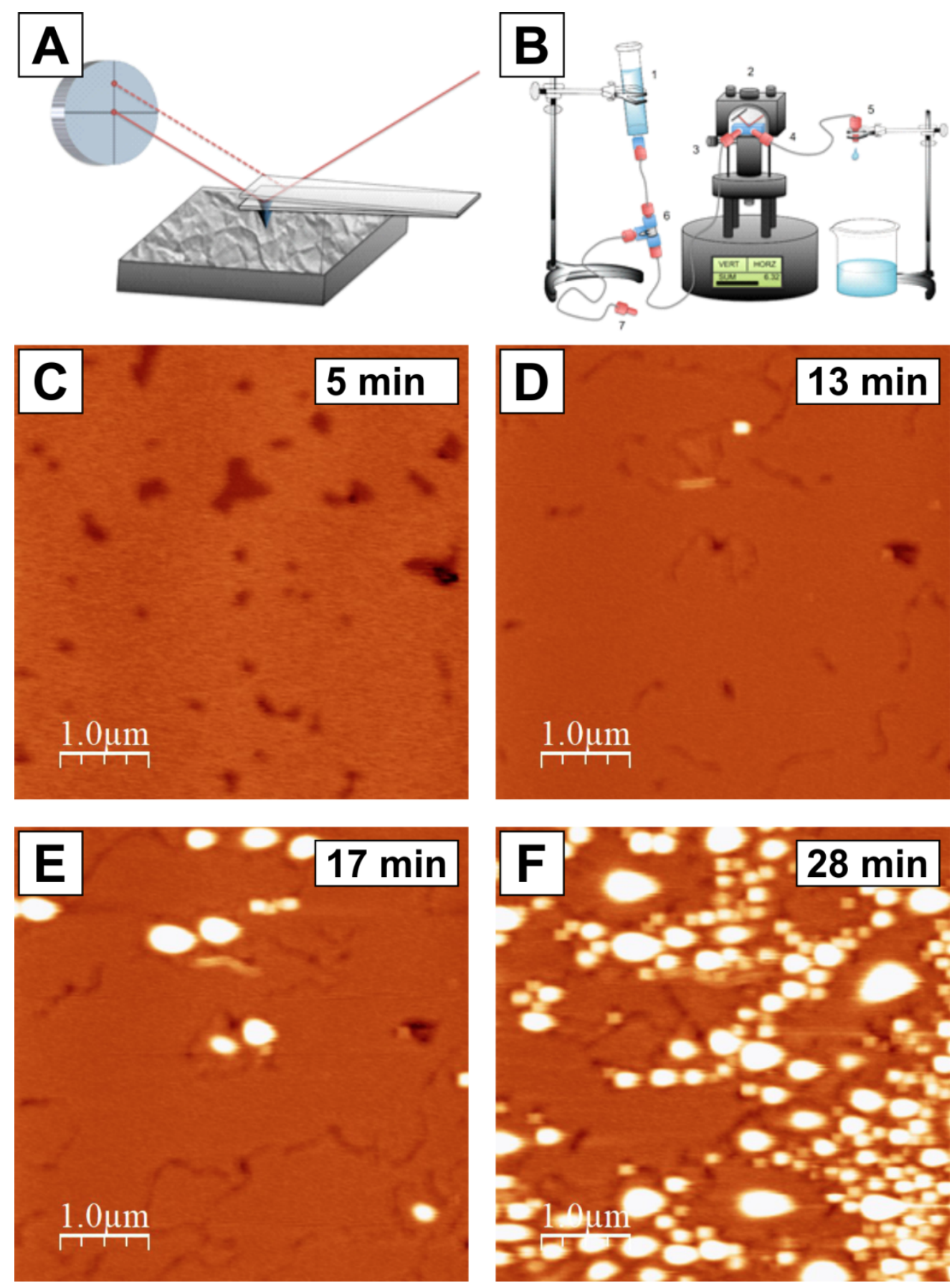

Figure 9. The principle of AFM (A) and the set up that allows continuous flow measurements (B) [91]. In the example, images are shown for an SLD composed of 5:3:2 by molar ratio DPPC:POPC:POPG both before (C) and after its interaction with $1 \mu \mathrm{M}$ MLT in $50 \mathrm{mM}$ Tris buffer (pH 7.4). The interaction leads to an initial expansion of defects and disappearance of the fluid domains (lower part of D) combined with re-adsorption of MLT/lipid aggregates at the edge of the defects (upper part of $D$ ), followed by further removal and re-adsorption (E and $F$ ). 
Figure $9 \mathrm{C}-\mathrm{F}$ shows the time evolution for the exposure of $1 \mu \mathrm{M} \mathrm{MLT}$ under continuous flow to a DPPC:POPC:POPG SLB (5:3:2 by molar ratio) in $50 \mathrm{mM}$ Tris buffer. As a reminder, DPPC was included in this mixture in order to induce gel-fluid domains as well as defects for easier visualisation of the SLB. Shortly after exposure to MLT, the biomembrane was still essentially unaltered with some visible defects (dark spots) as well as smaller regions of fluid membranes (Figure 9C). After some minutes (Figure 9D), the action of MLT can be observed as a decrease in the number of defects, which convert into thin channels in regions of the fluid membrane. Shortly afterwards (Figure 9E), aggregates (bright spots) start to be observed at the edges of the defects. Their number increases continually over time until almost half an hour has elapsed (Figure 9F), by which time practically the whole SLB has been re-structured. It can be inferred that the continuous exposure of $1 \mu \mathrm{M}$ MLT to the SLB results in lipid removal and therefore fluidization (thinning) of the membrane, and the lipid molecules lost from the membrane can interact with peptide in solution leading to the adsorption of MLT-lipid aggregates. A strength of AFM, therefore, is to provide information on the lateral organisation of the interface, and in this case the highly heterogeneous nature of the interfacial processes taking place as a result of the MLT interaction is revealed. Such information is not accessible using other techniques where a macroscopic property is probed or lateral homogeneity is assumed in the data interpretation.

Brewster angle microscopy (BAM) is an alternative imaging technique used to reveal lateral inhomogeneities of biomembrane systems and their interactions at the air-water interface. When p-polarized light is directed at the air-liquid interface at the Brewster angle $\left(\sim 53^{\circ}\right.$ for water), the reflected light is negligible. Any change in the interfacial refractive index, e.g. due to the presence of a surface film, will induce light reflection which can be detected by a camera that provides real-time images of the interface [173]. BAM has the ability to image anisotropy, besides total differences in refractive index, and thus can provide quantitative structural analysis in addition to the qualitative optical information [174]. BAM imaging has been extensively used in studying monolayers or films at the airliquid interface of amphiphilic molecules, proteins, DNA, drugs or nanoparticles [175] and specifically in the morphological features of lipid monolayers [176], as well as in binding processes involving peptides or proteins $[48,177-180]$. In these latter studies, BAM has 
been able to provide or corroborate results of peptides interactions with lipid monolayers regarding either the monolayer composition [177], charge [48] or phase state [180], as well as peptide charge or hydrophobicity [179]. Specifically, Ciumac, et. al [48] used DPPC and dipalmitoylphosphatidylglycerol (DPPG monolayers) to mimic the charge difference between mammalian and bacterial cell surfaces to study the binding of a synthetic AMP with the general sequence $\mathrm{G}(\mathrm{IIKK})_{4} \mid-\mathrm{NH}_{2}$. Here, BAM images demonstrated the ability of the peptide to interact and influence the lipid lateral organization through a fluidizing effect that was more pronounced from DPPG than DPPC. BAM has also been shown to have the ability to distinguish the interaction processes of peptides with similar structure and charge but different degrees of hydrophobicity [179]. Results revealed that both a1- and a2- purothionin were able to disrupt the interfacial domain structure of the condensed phase DPPG monolayers removing lipid during this process and both penetrated the lipid monolayer in addition to adsorbing as a single layer to the lipid head group. In this case, BAM was able to help distinguish or corroborate the increase of surface pressure process for each peptide but also showed the preference of one of the peptides for the more hydrophobic edges between lipid domains.

\subsection{Determination of Interfacial Interactions with Specific Chemical Groups Using Infrared Spectroscopy}

Infrared (IR) spectra describe the chemical groups present within a sample based on wavelength specific absorption bands. Vibrational modes which cause a dipole change within chemical groups are IR active, with the absorbed radiation matching the groups vibrational frequency. A single chemical group can have multiple vibrational modes as long as these vibrations are associated with a dipole change ( $3 \mathrm{~N}-5$ for linear groups and $3 \mathrm{~N}-6$ for non-linear, where $\mathrm{N}$ is the number of atoms).

Attenuated-total reflectance Infrared spectroscopy (ATR-FTIR) allows for spectroscopic examination of the changes in chemical composition at solid interfaces. In this experimental setup an IR beam is internally reflected, often multiple times, through 
an IR transparent solid substrate (zinc selenide, KRS-5, Diamond, silicon and germanium are commonly used). An evanescent wave is formed at the interface during each reflection. The IR beam penetrates up to several microns from the solid surface with exponential decay intensity from the interface (a schematic of this process is shown in Figure 10A). Changes in the chemical composition at or close to the interface can be monitored by the changes in absorption spectra.

ATR-FTIR has been used to examine SLBs deposited predominantly at germanium interfaces [181]. The technique is versatile and the resolved experimental data can provide information not only on the chemical composition but also on lipid phase states [80], time dependent changes in composition, the secondary structure of proteins and peptides [182], and (if coupled with a polarizer) molecular orientations relative to the interface [114]. Thus, ATR-FTIR can be a powerful technique in examining biophysical interactions with SLBs. Measurements are usually conducted with $\mathrm{D}_{2} \mathrm{O}$ instead of $\mathrm{H}_{2} \mathrm{O}$ in the bulk solution. This is because the $\mathrm{H}_{2} \mathrm{O}$ bending mode occurs at $1645 \mathrm{~cm}^{-1}$, which is the same region as the amide I (carbonyl) stretch of polypeptides and near to many other useful IR modes for protein lipid interactions such as carboxylic acids [183]. Such a coincidence can make resolution of these peaks difficult in the very low signal spectra measured for single SLB samples. Conveniently the $\mathrm{D}_{2} \mathrm{O}$ bending mode occurs away from this region at $1215 \mathrm{~cm}^{-1}$ [183]. 

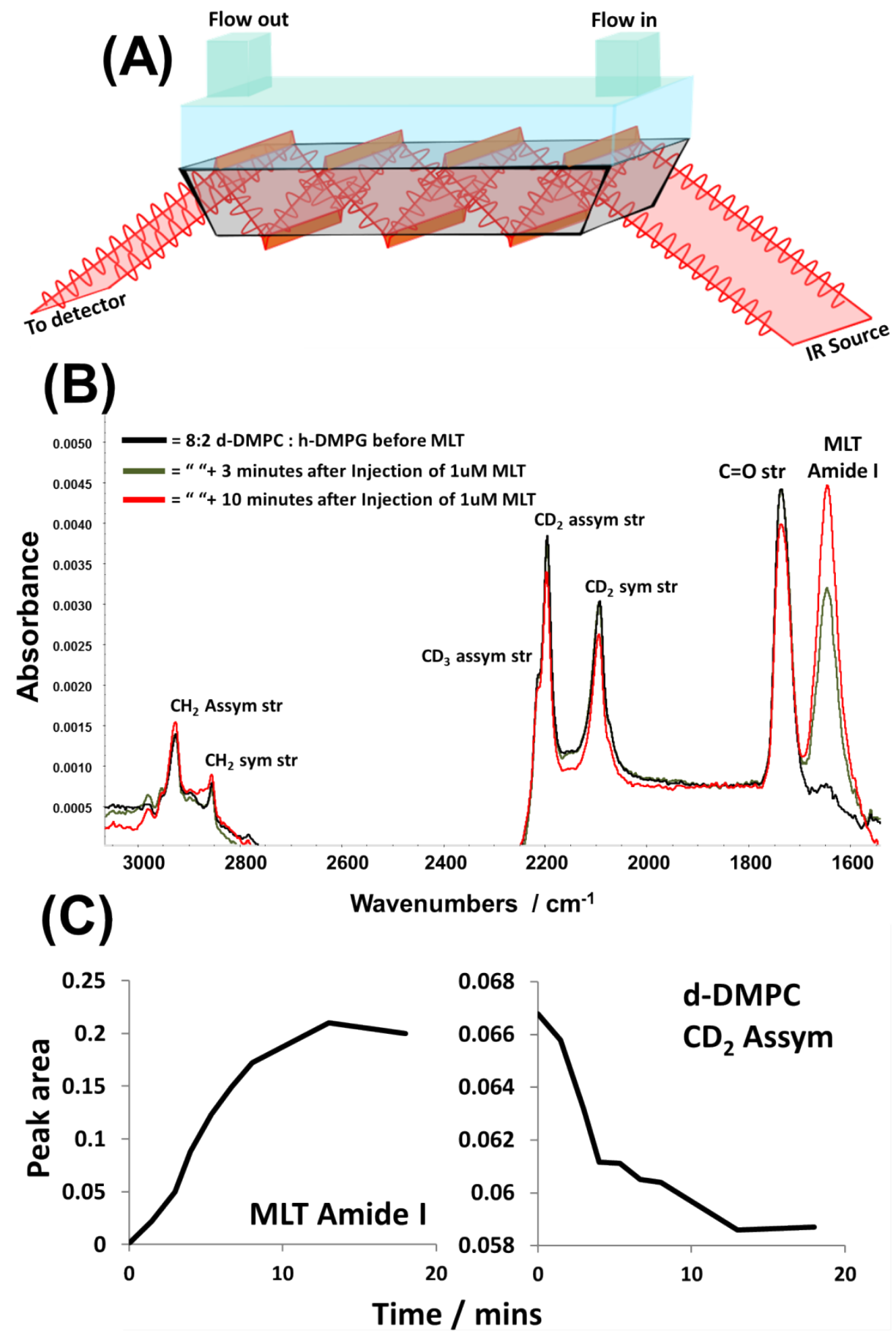

Figure 10. A schematic (not to scale) showing the total internal reflection of an IR beam through an IR-transparent prism (A); note the evanescent waves produced at points of total internal reflection. In the example (B), spectra are shown for a partially chain-deuterated SLB composed of 8:2 dDMPC:h-DMPG in molar ratio both before (black) and after (grey and red) its interaction with $1 \mu \mathrm{M}$ MLT in $50 \mathrm{mM}$ Tris buffer (pH 7.4), which is passed through the measurement cell. Note the differential C-H and C-D stretches from DMPG and DMPC, respectively, and the appearance of the 
Amide I band to the spectra upon MLT binding to the biomembrane. The changes in the Amide band from MLT and the $C_{2}$ asymmetric band from the DMPC vs. time after the introduction of the peptide into the flow cell are given (C).

The IR signal from the surface components is typically very low in intensity when examining the interactions of species from solution with SLBs. The analysis is mainly conducted using the strong IR bands in the functional group region ( 1550 to $\left.4000 \mathrm{~cm}^{-1}\right)$. The principle IR bands of interest for phospholipids are the aliphatic chain stretches (both symmetric and asymmetric see Figure 10B) and the carbonyl stretch from the ester groups which attach the acyl chains to the head group. Changes in the position and width of the aliphatic chain stretches can inform on changes to the local lipid environment [80]. Changes in the intensity of the adsorption bands informs on a relative changes in the material at or close to the bulk interface [115].

Selective deuterium labelling can be used to differentiate membrane components in FTIR measurements. Figure 10B shows ATR-FTIR spectra for an SLB composed of 8:2 dDMPC:h-DMPG in molar ratio on a silicon support immersed in $\mathrm{D}_{2} \mathrm{O}$ buffer solution (black line), where the prefix ' $d$-' refers to chain-deuterated lipid and prefix ' $h$-' refers to normal hydrogenous lipid. The labelling of the sample yields spectra where the IR absorption bands for the acyl chains of each lipid type are separated into C-D and C-H stretching modes. This enables the individual examination of each lipid species in the mixed bilayer. The exposure of $1 \mu \mathrm{M}$ MLT passed through the ATR flow cell causes the Amide I band of the peptide to appear in the region between 1600 and $1700 \mathrm{~cm}^{-1}$ (Figure 10B). This is the strongest adsorption band from polypeptides and is primarily composed of carbonyl stretches from the amide backbone. The amide I band is often broad as it has contributions from amide carbonyls from differing secondary structural motifs $[115,182,183]$. If the experimental spectra are of high quality the relative secondary structural components within polypeptide may be identified using curve fitting procedures [182]. Therefore, changes in polypeptide secondary structure before and after membrane binding maybe resolved. 
As the concentration of MLT used in the example is too low for the peptide dissolved in solution to contribute to the recorded spectra, the appearance of this band can therefore be attributed to the accumulation of peptide at or near the solid-liquid interface, in this case due to the interaction of the peptide with the SLB. The increase in the Amide I band was concurrent with a decrease in the $C_{2}$ asymmetric stretching peak intensity from $d$ DMPC (Figure 10C). This reveals that peptide accumulation in the interfacial layer was associated with a decrease in lipid content of the biomembrane, in this case the DMPC component. The contribution of aliphatic stretches from the peptide itself make the $\mathrm{C}-\mathrm{H}$ stretching region difficult to interpret and therefore the potential loss of DMPG form the membrane was not resolved.

Infrared reflection-absorption spectroscopy (IRRAS), which is also known as external reflection Fourier transform Infrared (ER-FTIR) spectroscopy, is a surface-sensitive technique similar to ATR-FTIR which can be employed to examine systems and interactions involving monolayers at the air-liquid interface. In this technique, a mirror assembly reflects an IR beam onto the air-liquid interface at a set angle of incidence and the beam reflected from this interface is examined as a function of wavenumber. Any IR active functional group present at or close to the interface will be identified in the spectra due to its distinct absorption bands, allowing for chemical identification of the interfacial constituents [184]. Like ATR-FTIR, information on the orientation of molecular species at the interface can be derived through the comparison of the absorption bands obtained from different polarizations of the incident IR beam ( $S$ and $P$ ). Examples of the use of this technique in membrane interaction studies include comparing of the membrane disruptive activity of a range of natural antimicrobial peptides [115], the interaction of host-defence proteins with model bacterial surfaces [185] and the cation mediated interactions of ribonucleic acids with membrane surfaces [186].

\subsection{Resolution of the Interfacial Structure and Composition by Neutron Reflectometry and X-ray Reflectometry}

Neutron reflectometry (NR) is a large scale facility technique used in the structural analysis of thin films at or close to bulk interfaces. Neutrons, as their name suggests, are neutral in charge and therefore only interact with the atomic nucleus. Neutron beams can, 
therefore, penetrate deeply into samples as the nucleus occupies a minute volume of an atom, samples buried inside complex environments can be probed, such as the solidliquid flow cells commonly used in SLB studies. Cold, thermal and epithermal neutrons have wavelengths in the Ångström regime, making them suitable probes of molecular structure and energies close to or the same as air at room temperature meaning they are non-damaging to biological samples. Lastly, the neutron scattering magnitude (known as scattering length, b) is non-monotonic across the periodic table, and even different isotopes of the same element can have strongly differing scattering lengths. Most usefully the two most common isotopes of hydrogen, protium (99.98\% natural abundance) and deuterium ( $0.02 \%$ natural abundance) have strongly differing neutron scattering lengths. As a result of this, values of the neutron scattering length density (SLD, equivalent to the scattering length of a molecule divided by its molecular volume) are widely separated for $\mathrm{D}_{2} \mathrm{O}$ (relative high value) and $\mathrm{H}_{2} \mathrm{O}$ (relatively low value), in contrast to optical refractive indices where the respective values are much more similar. The SLD of the biological macromolecules in their natively labelled forms is between that of $\mathrm{H}_{2} \mathrm{O}$ and $\mathrm{D}_{2} \mathrm{O}$. By mixing $\mathrm{H}_{2} \mathrm{O}$ and $\mathrm{D}_{2} \mathrm{O}$ as the solvent, the SLD of the bulk solution can be matched to that of individual components within a complex biological sample, which is a technique that can be exploited in order to emphasize the scattering of another. By building up a series of data sets obtained under differing solution isotope conditions (termed contrasts) the relative distribution of components within a biological complex can be resolved [187]. When a neutron beam is reflected at a layered interface, interference can occur between waves reflected at the top and at the bottom of an interfacial film (Figure 11A), which gives rise to an interference pattern (Kiessig fringes) in the reflectivity profile [188]. The spacing and intensity of the interference fringes yields structural information on the thickness and SLD of any layered material present at the interface. The experimental reflectivity data describe the SLD profile across the interface, which can be used to model the interfacial structure. The data can be analysed in several different ways, but model data fitting of the thickness, composition, solvation and roughness of a finite number of stratified layers parallel to the interface is by far the most common.

SLBs and monolayers, due to their interfacial nature, lend themselves to structural study by NR [189]. This technique is often used to examine the structure of and structural 
changes to model membranes that have been challenged with a difference in condition or an interacting agent. Hydrogen/deuterium labelling of both the samples and solution is used to highlight the relative distribution of components in or adjacent to the model membrane. Lipid deuterium labelling is commonly employed to resolve both the internal distribution of lipids within a membrane [74,84] and allow for easy differentiation of lipid and polypeptide components across the membrane structure after interactions from solution species take place [190]. The mechanism by which an agent interacts with a membrane can be resolved structurally and with precision using NR [62,191], making this a useful tool for examining membrane biochemistry. 

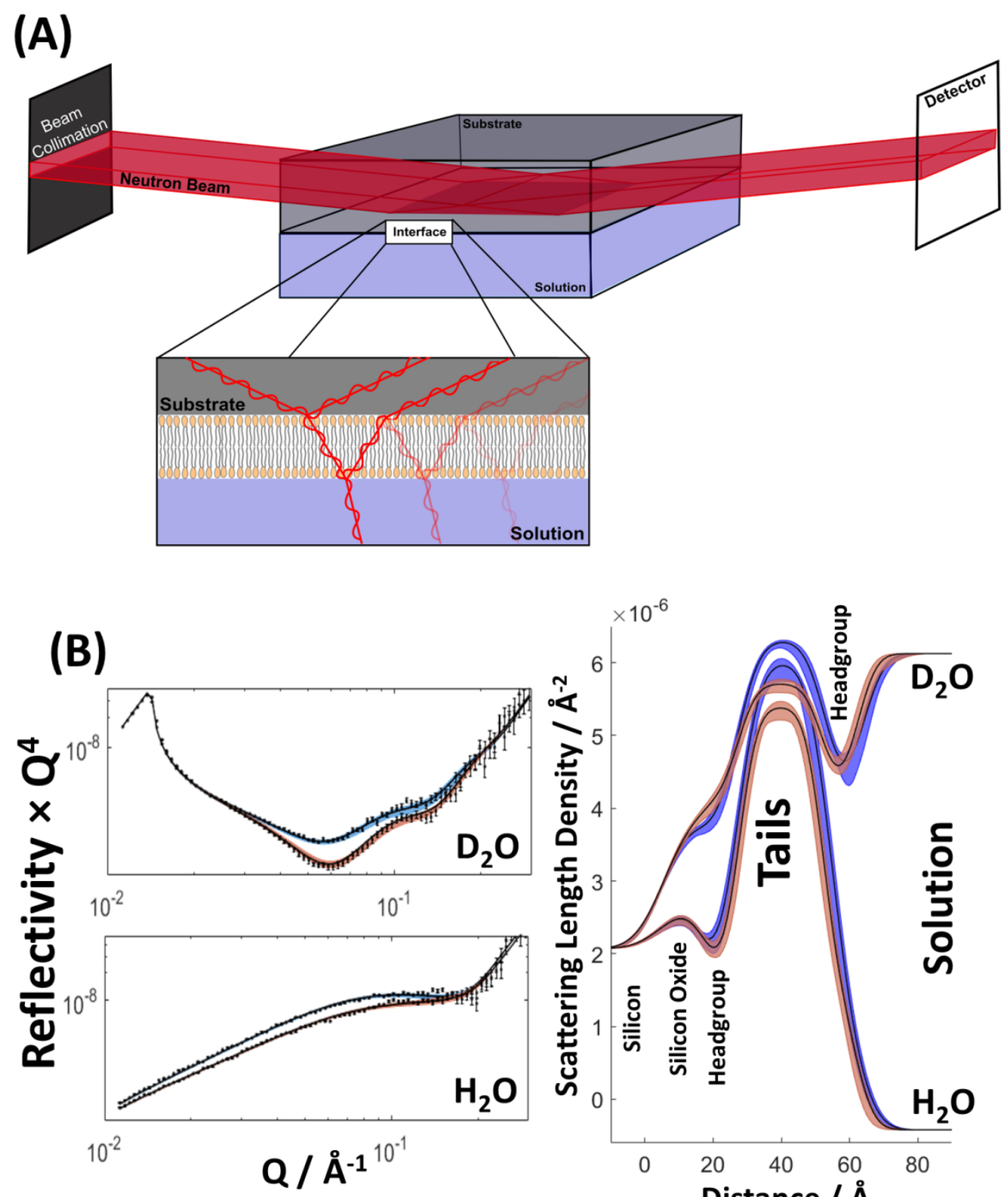

8:2 (w/w) d-DMPC : d-DMPG Bilayer before MLT

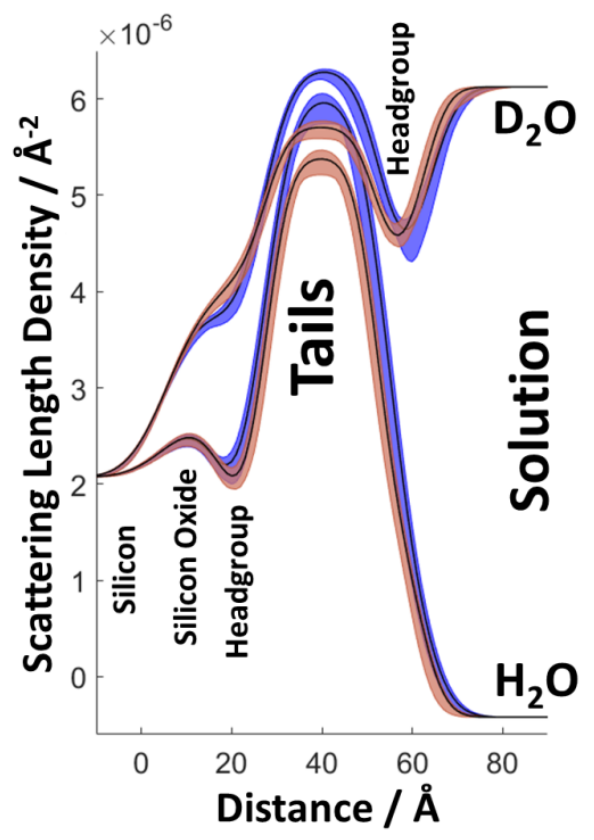

Figure 11. A schematic representation of the application of NR at the solid-liquid interface (A). An incident beam of neutrons passes through a solid substrate (usually silicon or quartz) virtually unperturbed before reflection at the interface. The proportion of reflected and refracted neutrons is determined by the angle of incidence, neutron wavelength and the interfacial composition. Any layered material at the interface, such as SLBs, will result in reflection at each interface, and interference between these reflections gives rise to Kiessig fringes, manifested as undulations in the data whose frequency and intensity is directly related to the thickness of the layer and its composition. This technique is highly sensitive to the type of hydrogen isotopes present in the 
sample, which can be exploited to resolve the structure and composition of complex biomacromolecular assembles. In the example (B), data are shown for a chain-deuterated SLB composed of 8:2 (w/w) d-DMPC:d-DMPG both before (blue) and after (red) its interaction with $5 \mu \mathrm{M}$ MLT in $50 \mathrm{mM}$ Tris buffer (pH 7.4), which is passed through the measurement cell. The use of hydrogen isotope labelling of the lipids and solution allows the position of the peptide and relative structural changes of the membrane to be resolved.

In Figure 11B example NR data from a silicon water interface coated with an SLB of 8:2 DMPC:DMPG is shown (blue data). The acyl chains of the phospholipid bilayer are deuterium labelled in order to differentiate them easily from the interacting MLT. The bilayer was measured in two solution contrasts, $\mathrm{H}_{2} \mathrm{O}$ and $\mathrm{D}_{2} \mathrm{O}$. The $\mathrm{D}_{2} \mathrm{O}$ data set is sensitive to the contribution of the lipid head groups and peptide to the interfacial structure due to the difference in SLD between these hydrogenous components and the deuterated solution. The $\mathrm{H}_{2} \mathrm{O}$ contrast is sensitive to the deuterated acyl chains of the lipid due to the large difference in SLD between the components. The resolved SLD profile (Figure 11B right) reveals that prior to the interaction of the MLT there is a high coverage bilayer ( $96 \%$ of the sampled surface) at the silicon water interface with the lipids having an average molecular area (derived from experimental data fitting) of $53.2( \pm 2) \AA^{2}$, head group thicknesses of $9( \pm 1) \AA$ and chains thickness of $27( \pm 1) \AA$. Upon the interaction of MLT ( $5 \mu \mathrm{M}$ in the solution), changes were noted in the interfacial structure. The SLD of the acyl chain region of the interfacial structure decreased (Figure 11B), which model data fitting suggested was due to the removal of lipids and the incorporation of MLT into the bilayer chains. Indeed, an $18( \pm 2) \%$ exchange of lipid for peptide in the chain region of the membrane at equilibrium protein binding was observed, as was a slight thinning of the bilayer, probably as a result of lipid removal during the MLT interaction. From these data, we have gained structural and mechanistic insights into the antimicrobial activity of MLT against the model bacterial membrane. Results suggest that the peptides mechanism of antimicrobial activity against the model Gram negative membrane involves lipid removal and penetration of significant amounts of peptide into the acyl chain region of the lipid membrane.

The application of NR at the air-liquid interface is analogous to that at the solid-liquid interface with the exception of a new approach that has been developed to resolve the 
composition of binary mixtures more accurately and much faster than was previously possible [192]. Maximum neutron flux is placed on the sample at low $Q$ in two isotopic contrasts of the interfacial material but both in air contrast matched water (zero SLD); structural information at higher $Q$ is discarded. The measurements are used to calculate the interfacial amounts of each component. This approach removes certain ambiguities of using a structural model where parameters such as interfacial roughness are often not independently resolved. The increase in time resolution of measurements has been estimated to be around a factor of 60 [193]. The approach has been applied to good effect in studies of the interactions of a short designed antimicrobial peptide ( $\left.\mathrm{G}(\mathrm{IIKK})_{4}-\mathrm{I}-\mathrm{NH}_{2}\right)$ with lipid monolayers $[48,194]$. Kinetics of interaction of the peptide as well as loss of lipid from the model membrane were revealed in real time for the first time to resolve effects of lipid charge and degree of saturation as well as the starting surface pressure on the rate and extent of interactions.

$X$-ray Reflectometry (XRR) is more frequently used to investigate lipid monolayers at the air-liquid interface due to the dominating scattering from solid substrates over the weak scattering of the lipid components. However, it is possible to study SLBs at the solidliquid interface with the $X$-ray beam traversing the liquid [195]. XRR is a good complement to NR, since it provides a larger dynamic range and - in part due to the higher flux synchrotron sources available - better signal-to-noise than NR. However, X-rays are ionising and can lead to sample damage, while higher absorption cross sections can limit the penetration depth of the [196]. At the air-liquid interface, X-rays, like neutrons, reflect at the free water surface, and the reflected beam is modulated by the relative refractive index difference between air, the thin film and the bulk liquid [197-199]. An example of how XRR can be used to investigate the effect of AMPs on different model membranes can be found in the work of Travkova and co-workers [200]. From the electron density profile they could locate the peptide in a POPG monolayer, with a partial penetration in the chain region together with adsorption to the head group. The interaction with DPPG gave a different result: the location of the AMP was at the boundary between the head groups and the bulk solvent, suggesting that no penetration in the lipid layer had occurred. From this work, it is clear that not only the extent of peptide binding but also its colocalisation within the lipid monolayer can be revealed using XRR. Finally, grazing angle 
X-ray diffraction and X-ray fluorescence are other popular techniques used to study biomembranes at the air-water interface in terms of their in-plane molecular packing and the quantification and distribution of specific chemical elements and ions, respectively [201-203].

\section{Complementarity of Surface-Sensitive Techniques to Reveal the Interaction Mechanisms of an Antimicrobial Peptide with Model Biomembranes}

\subsection{Complementary Comparison of Analytical Data}

Since not only the principle and sensitivity of the technique applied but also the properties of the membranes and experimental conditions used largely influence the experimental outcome, it is quite challenging to make a systematic comparison based on reports from the literature. Therefore, in this review, we have presented new data on the effects of MLT on SLBs and lipid monolayers composed of mixtures of PC and PG lipids at molar ratios relevant for Gram negative membranes under similar experimental conditions - 50 $\mathrm{mM}$ Tris buffer $(\mathrm{pH} \mathrm{7.4)}$ with the lipid membrane in the fluid phase at room temperature and using a number of bench-top and large facility analytical techniques. MLT is one of the most studied AMPs and a search on the web of Science at the time of submission using "Melittin" and either "supported lipid bilayer" or "planar lipid bilayer" gives a total of 82 references during the last 2 decades. If instead "lipid monolayer" or "langmuir monolayer" is used then 35 references are found since 1992. MLT does not display selectivity against a specific membrane beyond that of Gram positive cells. A full review of the activity of MLT is beyond the scope of this review (which is methodologically focussed) but a useful discussion of this topic can be found in the review of MLT by Raghuraman and Chattopadhyay [204]. In this review, the interactions of the peptide were examined using surface pressure and ellipsometry measurements on a Langmuir trough as well as QCM-D, ellipsometry AFM, ATR-IR and NR measurements on SLBs to highlight the use of a complementary analysis approach in deciphering the nature of membrane interactions with molecular precision. The reader may have noted the strong coherence of the experimental results across each technique in addition to the differing nature of the information afforded by each one. 
MLT adsorption to a model SLB was confirmed by ATR-IR since the amide band is clearly seen in the spectra (Figure 10). Both QCM-D and ellipsometry exhibited maximum values of the interfacial mass, respectively (Figures $7 D$ and $8 D$ ), before rearrangement of the interfacial material resulted in loss. These data provide an indication that the interaction mechanism under consideration is more involved than simple peptide binding. Additionally, MLT exposure to a pre-compressed lipid monolayer resulted in an initial increase in surface pressure and change in $\Delta$ (itself related to the overall interfacial excess) before the surface pressure relaxed while the change in $\Delta$ did not (Figure 8F). Together these data hint that lipid is lost from the surface monolayer, the molecular packing of which strongly affects the surface pressure, while peptide continues to bind to the interfacial structure in some way.

Uniquely, AFM data suggest that MLT preferentially binds to SLB defects and that this initial binding leads to SLB restructuring leaving behind a highly heterogeneous interfacial film (Figure 9C-D). Moreover, aggregates appeared on the membrane surface (Figure $9 \mathrm{E}-\mathrm{F}$ ) that probably involve peptides in complexes with lipids lost from the SLB. It may be possible that such aggregates lead to the sharp increase in $\Delta$ observed in the ellipsometry data for the lipid monolayer at the air-liquid interface (Figure 8F). Indeed, lipid removal due to the interaction of MLT with SLBs is the main source of mass loss from the biomembrane. This point was confirmed both by NR (Figure 11), which is sensitive to the composition of layers modeled normal to the interface, and ATR-IR (Figure 10), where a decrease in a $C_{2}$ stretching peak demonstrated the loss of the deuterated lipid (dDMPC) from the interface.

$N R$, due to its unique capability in resolving complex structures, showed that MLT incorporates into the hydrophobic environment of the SLB. It is likely that this intercalation of peptide into the membrane drove the loss of lipid from the interface, which in turn resulted in the binding of MLT-lipid aggregates to the membrane defects, as observed with AFM. Even so, the results from these two techniques are not fully consistent, as the aggregates were observed with AFM but not with NR. This type of discrepancy can be attributed to differences either in sample handling during the experiments or in the sensitivities of the techniques. It is possible that differences in the flow cell geometry and surface-to-volume ratio between the two experimental setups favoured aggregate binding 
for AFM but not for NR. Indeed, the surface exposed to the MLT solution was less than 1 $\mathrm{cm}^{2}$ in AFM (also: QCM-D and ellipsometry) while it was $\sim 30 \mathrm{~cm}^{2}$ in NR, and typically 1$2 \mathrm{~mL}$ of solution was flowed for AFM (also: QCM-D) while typically $\sim 10 \mathrm{~mL}$ was flowed for NR (also: ellipsometry). Nevertheless, it is highly likely that the surface density, or area coverage, of lipid and peptide in the aggregates was simply below the detection limit of NR.

Lastly, it may be noted that the SLB structural rearrangement into a heterogeneous layer driven by the intercalation of the peptide into the membrane and the subsequent lipid removal process was also suggested by QCM-D, due to a change in the overtone dissipation and frequency spreading at its final state as compared to that of the initial bilayer (Figure 7D), and to some extent by ellipsometry, through a reduction in the layer density (Figure 8D).

In conclusion, from these results we can present a mechanism where from solution MLT binds to and penetrates the model biomembrane, which results in its fluidization and thinning due to lipid loss, followed by binding of MLT-lipid aggregates from solution. The combined analysis approach used here was able to provide precise molecular level insight into the antibacterial activity of MLT. No single technique was able to give a complete overview of the processes occurring, yet by comparing and contrasting results from multiple sources both an overview of the nature of the interaction as well as information on the interaction mechanism were obtained. This understanding of the molecular rearrangement of the model membrane upon MLT interaction can now be elaborated with results obtained from in vitro and in vivo studies in order to gain a complete understanding of these complex biological processes.

Figure 12 shows a schematic of the final picture of the mixed MLT-biomembrane structures, and serves to highlight the different sensitivities of the techniques reviewed. From the use of the biomembrane interaction example presented, it is hoped that the reader can gain an understanding of how results from one technique feed into and supplement the understanding of results from another, yielding a more thorough interpretation and thus a greater understanding of the underlying scientific problem. 


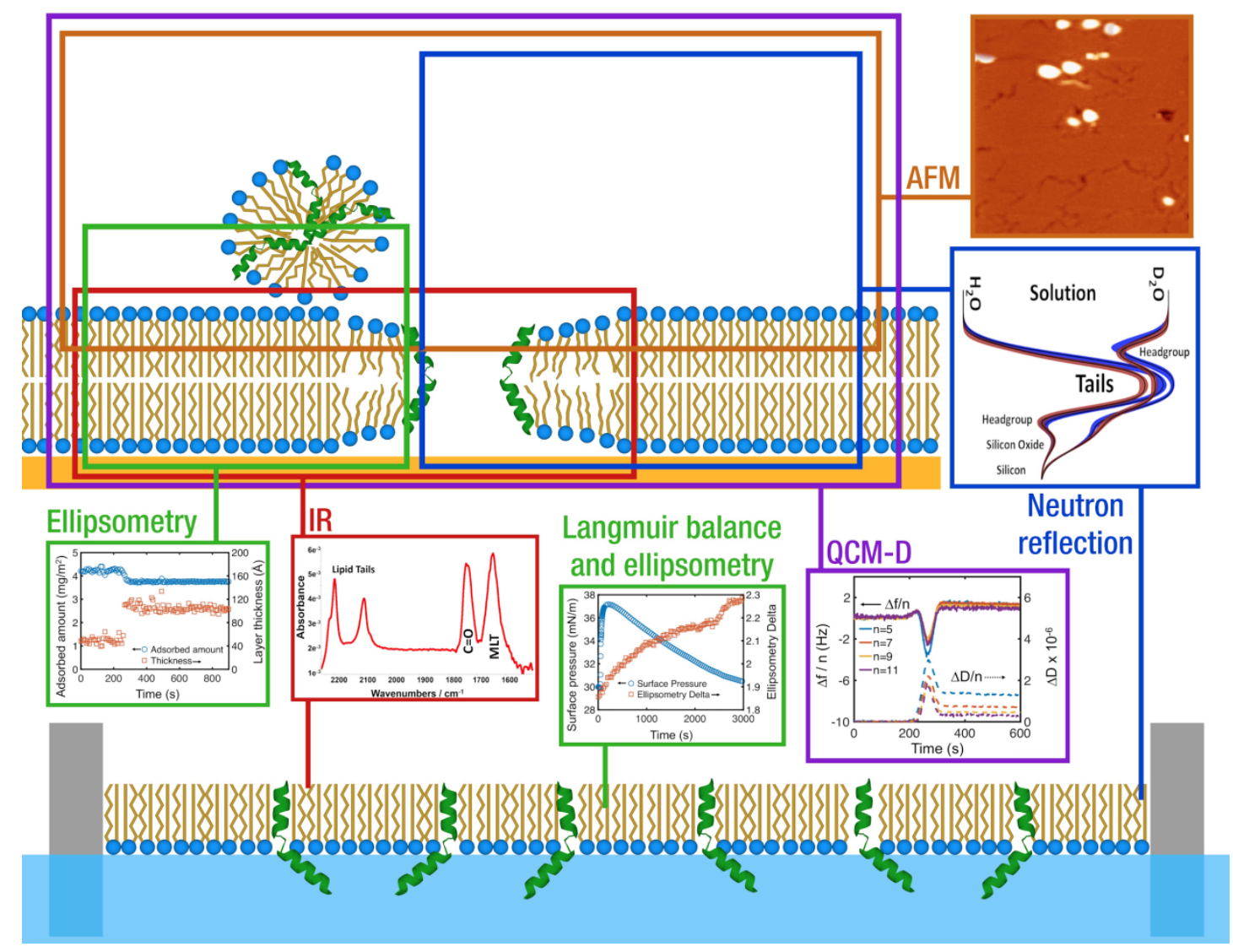

Figure 12. Schematics of the final interfacial structures upon MLT interactions with PC:PGbased model membranes: an SLB at the solid-liquid interface (top) and a lipid monolayer at the air-liquid interface (bottom). QCM-D, ellipsometry, IR and NR are techniques that average over the entire interface structure while AFM is unique at capturing the morphology of the membrane surface. QCM-D and ellipsometry provide indications on the total mass changes and overall membrane structure (either the total thickness or the level of homogeneity and softness of the membrane), while IR gives information on changes occurring at the level of specific chemical groups. NR gives the location and composition of the film but its sensitivity is lacking when the surface coverage is low (as perhaps for the MLT-lipid aggregates present on top of the original lipid bilayer observed by AFM). Monolayers at the air-liquid interface can be studied using the Langmuir trough in combination with techniques such as ellipsometry, IR and NR, as well as BAM and XRR which were not considered in detail in the present work.

\subsection{Critical Comparison of Analytical Techniques}


In the consistent example given above, many techniques have yielded similar information (e.g. peptide interactions were implied, albeit in differing ways and with differing sensitivity, by all of the techniques) while others were unique in their information content. For example, the location of MLT in the biomembrane was revealed only by NR, and the presence of membrane surface aggregates was observed only by AFM, although a hint of such a secondary interaction was provided by ellipsometry at the air-liquid interface as well. Each technique used in this model membrane study has distinct advantages and limitations in the examination of biomolecular interactions. Table 1 provides for each technique applied in the present work descriptions of i) the information content provided, ii) the typical nature of the analysis, and iii) details of the information afforded for our chosen example of MLT interactions with model PC:PG-biomembranes.

Surface pressure measurements on a Langmuir trough, and the application of QCM-D or ellipsometry, are perhaps the most straightforward techniques to employ on a new system in terms of experimentation and elementary data interpretation. Therefore they are suitable for determining macroscopic changes occurring at model biomembranes by screening a large number of interactions under different physical conditions (lipid composition, concentration, ionic strength and $\mathrm{pH}$ ). Even so, the challenge of producing stable monolayers that contain unsaturated lipids should not be underestimated, and the extent to which the data analysis is taken will determine the depth of information accessible. For example, a straightforward Sauerbrey model in QCM-D may provide a basic estimate of the change in wet mass of a rigid film, but for elastic films a more complex Voigt model may be required. Also, ellipsometry data at the air-liquid interface may be left simply as 'change in $\Delta^{\prime}$ ' or a model may be applied that takes into account different refractive indices of species as well as anisotropy.

AFM and NR give probably the most detailed information accessible, and can be used to examine the structure and morphology of a model membrane before and after interactions with species from solution, albeit in different and highly complementary geometries. IR techniques make a valuable addition to any such study as, unlike the other bench-top techniques, it allows the molecular complexity of the model biomembrane to be resolved in terms of the physical state surrounding the chemical groups present. NR also provides information on the biomembrane structure, but also yields the distribution of components 
normal to the interface, which includes composition, solvation and roughness. Both NR and IR significantly benefit from deuterium labelling of the samples and solvent, but this requires both expertise to employ and additional experimental costs. Another important aspect to consider is that access to NR is only available at national and international large facilities and requires a significant amount of acquired expertise to undertake in order to gain the large depth of information accessible. Another limiting factor can be the volume required of a species interacting from the solution with a lipid monolayer on a Langmuir trough, which is typically significantly larger that required in flow cells used for techniques applied at the solid-liquid interface.

It is, therefore, the recommendation of the authors that any new investigation of a biomolecular interaction mechanism with model biomembranes is best started with the most straightforward and easily deployed techniques available, and the analytical complexity of the techniques used in the study and/or the data analysis approach can be developed during the investigation. Samples that show weak or transient membrane interactions can be screened via this method, and the parameter space of experimental variables can be refined, resulting in only the most viable samples taken forward for experiments using the more involved analytical techniques. This strategy allows for efficient use of the researcher's time and resources. Which set of techniques is best employed in a given study is dependent on the nature of that study. An interaction, for example, where the biomembrane is fully removed may not be ideally suited to NR and could easily be resolved using a combination of QCM-D, ellipsometry and/or IR where the loss of material in time can be detailed. Conversely, an interaction where a protein fully or partially embeds into model membrane may be optimally studied using $N R$ in combination with bench-top techniques such as a Langmuir trough, IR and/or AFM. Using this combination of techniques, it is a realistic aim to resolve the relative position, orientation and distribution of the components as well as any phase changes in the lipid as a result of this interaction.

Table 1. A comparison of the surface analytical techniques used here to elucidate the mechanism of MLT antibacterial activity. 


\begin{tabular}{|c|c|c|c|}
\hline Technique & Information Content & Nature of analysis & $\begin{array}{l}\text { Result from MLT/PC:PG bilayer } \\
\text { Interaction }\end{array}$ \\
\hline QCM-D & $\begin{array}{l}\text { Interfacial wet mass and } \\
\text { viscoelasticity }\end{array}$ & $\begin{array}{l}\text { Raw data interpretation } \\
\text { or model data fitting }\end{array}$ & $\begin{array}{l}\text { MLT adsorbs to the SLB and } \\
\text { surface rearrangement occurs }\end{array}$ \\
\hline $\begin{array}{l}\text { Ellipsometry } \\
\text { (SLBs) }\end{array}$ & $\begin{array}{l}\text { Interfacial mass, layer } \\
\text { thickness and density }\end{array}$ & Model data fitting & $\begin{array}{l}\text { MLT interaction increases } \\
\text { thickness and decreases the } \\
\text { density of the SLB }\end{array}$ \\
\hline $\begin{array}{l}\text { Langmuir } \\
\text { Trough }\end{array}$ & Monolayer packing & Raw data interpretation & $\begin{array}{l}\text { MLT adsorbs but relaxation } \\
\text { suggests looser monolayer } \\
\text { packing with time }\end{array}$ \\
\hline $\begin{array}{l}\text { Ellipsometry } \\
\text { (monolayers) }\end{array}$ & $\begin{array}{c}\text { Interfacial mass } \\
\text { combined with anisotropy }\end{array}$ & $\begin{array}{c}\text { Raw data interpretation } \\
\text { or model data fitting }\end{array}$ & $\begin{array}{c}\text { MLT adsorbs with a hint of a } \\
\text { secondary interaction process after } \\
\text { some time }\end{array}$ \\
\hline AFM & $\begin{array}{l}\text { In-plane structure } \\
\text { and morphology }\end{array}$ & $\begin{array}{l}\text { Raw data interpretation } \\
\text { or model data fitting }\end{array}$ & $\begin{array}{l}\text { MLT changes SLB morphology } \\
\text { and aggregates are observed on } \\
\text { the membrane surface }\end{array}$ \\
\hline ATR-FTIR & $\begin{array}{c}\text { Chemical group } \\
\text { composition, lipid phase } \\
\text { and polypeptide } \\
\text { secondary structure. } \\
\text { Peptide orientation with } \\
\text { polarization }\end{array}$ & $\begin{array}{l}\text { Raw data interpretation } \\
\text { or model data fitting }\end{array}$ & $\begin{array}{c}\text { MLT binds to the SLB is lipid is lost } \\
\text { from the interface }\end{array}$ \\
\hline NR & $\begin{array}{l}\text { Structure across the } \\
\text { interface and relative } \\
\text { positions of components }\end{array}$ & Model data fitting & $\begin{array}{l}\text { MLT removes lipid and penetrates } \\
\text { the chain region of the SLB } \\
\text { inducing its thinning }\end{array}$ \\
\hline
\end{tabular}

\subsection{The relevance of the lipids choice and experimental conditions}


As already mentioned, it is out of the scope of this review to give a comprehensive literature overview on the mechanism of action of MLT with model biomembranes. However, here we will link our results to some previous studies to highlight the importance of the experimental conditions specifically on the physical phase of the model biomembrane. In particular, AFM has been used to follow the effect of MLT on the morphology of SLB made of pure DMPC [110], pure DMPS [116] and pure DMPG [116] on gold made by Langmuir-Blodgett deposition. The morphology of the SLBs changed dramatically upon exposure to $10 \mu \mathrm{M}$ MLT at room temperature in $20 \mathrm{mM}$ Tris buffer $(\mathrm{pH}$ 7.5) enriched with $150 \mathrm{mM} \mathrm{NaCl}$ and $5 \mathrm{mM}$ EDTA: the initial adsorption of MLT to all types of SLB eventually led to partial solubilisation of the SLB made of DMPC and DMPG, but only restructured the SLB made of pure DMPS. Surprisingly, the kinetics of solubilisation was faster for DMPC than DMPG SLB, and this was attributed to the slowing down of the peptide reorientation and insertion in charged compared to zwitterionic membrane perhaps due to stronger electrostatic interactions with the lipid heads. Thus, it would seem that the lipid type has a dramatic effect on the final state of the SLB after the initial peptide adsorption and lipid removal. However, the melting temperature of DMPS [205] is 12-13 ${ }^{\circ} \mathrm{C}$ higher than DMPG [206] or DMPC [207] and therefore, the different mechanisms of action simply reflect differences in molecular packing and therefore in the fluidity [116] of the SLB. Therefore, it can be concluded from these studies that MLT is able to integrate faster into the fluid and uncharged SLB than into the stiffer and negatively charged SLBs. Indeed, the preference for lipids in the fluid phase rather than the gel phase was studied by Bodescu et al [111] using dioleoylphosphatidyl choline DOPC $\left(\mathrm{T}_{\mathrm{m}}=-16^{\circ} \mathrm{C}\right.$ [208]) and DPPC $\left(T_{m}=41^{\circ} \mathrm{C}\right.$ [209]) SLBs (1:1 molar ratio) on mica in the presence of $10 \mathrm{mM}$ Tris buffer ( $\mathrm{pH} \mathrm{7.6)} \mathrm{enriched} \mathrm{with} 80 \mathrm{mM} \mathrm{NaCl}$ at room temperature. In this case, incubation of $2 \mu \mathrm{M} \mathrm{MLT}$ solution led to the partial solubilisation of the DOPC rich, fluid phase and the typical formation of globular aggregates or mixed peptide-lipid micelles upon lipid removal. Together, these studies suggest that peptide adsorption is the initial step and that once a certain peptide concentration in the biomembrane is reached, lipids are removed, eventually leading to the formation of mixed peptide-lipid micelles: this is sometimes referred to as the mixed carpet-toroidal pore model of MLT action.

The reader may note that even though a consistent approach was attempted with the 
example of MLT interactions with PC:PG-based model biomembranes in the present work, in cases the choice of lipids used was adapted to specific requirements of particular techniques. For example, while POPC:POPG mixtures were the preferred lipid choice (usd in Langmuir trough, QCM-D and ellipsometry) since they better resemble the acyl chains present in cellular membranes, the commercial availability of deuterated lipids forced a different choice for the IR and NR experiments. In these cases, DMPC:DMPG mixtures were chosen as they could be readily purchased, and the experimental settings were chosen to conserve full fluidity of the system above the chain melting temperature of the lipid mixture. Also, DPPC was incorporated into the lipid mixture for AFM, since fluid SLBs made of POPC are featureless and form very quickly, making it very hard to know if a membrane has been formed at all during the experiment. Instead, islands of fluid membrane were shown to co-exist in a membrane that was mostly in the gel phase, and this aspect allowed observation of the preferential interaction of MLT with fluid phase membranes. It is therefore the opinion of the authors that in order to maximize the scientific insight from a biomolecular interactions project, the lipid composition of the biomembrane and the experimental conditions may be adapted accordingly, but the phase and fluidity of the biomembranes studied with various techniques should be strongly taken into account.

\section{Conclusions and Perspectives}

Langmuir monolayers at the air-liquid interface constitute the first model biomembranes ever produced and studied. Even though they are relatively simple with just one lipid leaflet, they allow control of the lipid packing and phase, and are therefore used conveniently to study biomolecular interactions. Supported lipid bilayers, on the other hand, are useful biomimics for rapid screening of the mechanistic aspects of molecular interactions with biomembranes that have two lipid leaflets. In order to improve the understanding of specific biomolecular interactions with lipids in a more relevant biomimetic system, more advanced membrane systems can be used where supported lipid bilayers are tethered to or float on top of the solid substrate. These are prepared themselves using lipid monolayers at the air-liquid interface. 
Through the application of complementary surface-sensitive techniques, detailed information on the molecular mechanisms by which molecules such as drugs, proteins and peptides interact with lipid membranes can be obtained. There are limitations of the use of model membranes platforms, such as a lack of natural fluctuations, unwanted interactions from an underlying solid substrate, and importantly reduced complexity from reality as specific proteins and receptors found in cell membranes are missing. Nevertheless, techniques that are sensitive to net changes in packing or interfacial mass (such as surface pressure isotherms on a Langmuir trough, ellipsometry or QCM-D), bilayer morphology (AFM or BAM), the presence of chemical groups (IR) and structure and composition (NR or XRR) of mixed systems represent a very powerful combination to unravel the mechanism of biomolecular interactions.

A consistent example of the interactions of the antimicrobial peptide Melittin with phosphocholine:phosphoglycerol-based model lipid biomembranes recorded under similar conditions has been used in the present work. This example was chosen in order to showcase the different information accessible from a suite of complementary surfacesensitive techniques, and to caution when the choice of lipids had to be adapted to meet the requirements of particular techniques. A coherent interaction mechanism was revealed, where peptide binds to and penetrates the model biomembrane, resulting in its fluidization and thinning from lipid loss, and peptide-lipid aggregates then bind from solution. It is intended that reference to the depth of information accessible from this example can assist the reader in important factors to take into consideration in the design of future studies of molecular interactions involving model biomembranes.

\section{Acknowledgements}

The idea behind this review was born though the planning of the PhD Summer Course Nanotools for Biomembranes (www.biomembranes.se), in Malmö, Sweden, in August 2019 where the techniques presented here were applied to study the interactions of a range of antimicrobial compounds with model membranes. The authors thank each of the fourteen students who participated with the data acquisition and helped improve and consolidate the ideas behind this review: Hameed Ahmed, Hanna Boyd, Ragna 
Guldsmed Diedrichsen, Skaidre Jankovskaja, Stephanie Jephthah, Erum Kakar, Mette Klitgaard, Katarzyna Makasewicz, Maxim Morin, Benedetta Petracca, Jadwiga Rambiesa, Johanna Utterström, Sarah Waldie and Yixuan Yan. The author also thanks other teachers involved in the course: Dr. Peter Falkman, Prof. Tommy Nylander and Dr. Nicolo Paracini. Some of the data were acquired prior and after the course: the authors thank the ISIS neutron source for granted beamtime to collect the NR data on MLT, L.A.C. thanks Mario Campana and John Webster for support with measurements on the SURF reflectometer, R.A.C. thanks Javier Carrascosa Tejedor for assistance with the monolayer measurements and Jayne Lawrence for use of the Kibron Langmuir trough and Accurion EP4 ellipsometer, and J.C.T. thanks Alberto S. Luviano for support with DPPC monolayer measurement. The authors also thank Biofilm - Research Center for Biointerfaces for financial support and Biolin Scientific for lending a trough during the course, and M.C. thanks the Swedish Research Council for financial support.

\section{References}

[1] Stefaniu C, Brezesinski G, Möhwald H. Langmuir monolayers as models to study processes at membrane surfaces. Adv Colloid Interface Sci 2014;208:197-213.

[2] Rascol E, Devoisselle J-M, Chopineau J. The relevance of membrane models to understand nanoparticles-cell membrane interactions. Nanoscale 2016;8:4780-98.

[3] Lösche M. Surface-sensitive X-ray and neutron scattering characterization of planar lipid model membranes and lipid/peptide interactions. Peptide-Lipid Interactions, vol. 52, Elsevier; 2002, p. 117-61.

[4] Rebaud S, Maniti O, Girard-Egrot AP. Tethered bilayer lipid membranes (tBLMs): interest and applications for biological membrane investigations. Biochimie 2014;107 Pt A:135-42.

[5] Yin H, Flynn AD. Drugging Membrane Protein Interactions. Annu Rev Biomed Eng 2016;18:51-76.

[6] Overington JP, Al-Lazikani B, Hopkins AL. How many drug targets are there? Nat Rev Drug Discov 2006;5:993-6.

[7] Singer SJ, Nicolson GL. The Fluid Mosaic Model of the Structure of Cell Membranes. 
Science 1972;175:720-31.

[8] van Meer G, Voelker DR, Feigenson GW. Membrane lipids: where they are and how they behave. Nat Rev Mol Cell Biol 2008;9:112-24.

[9] Simons K, Ikonen E. Functional rafts in cell membranes. Nature 1997;387:569-72.

[10] Oursel D, Loutelier-Bourhis C, Orange N, Chevalier S, Norris V, Lange CM. Lipid composition of membranes of Escherichia coli by liquid chromatography/tandem mass spectrometry using negative electrospray ionization. Rapid Commun Mass Spectrom 2007;21:1721-8.

[11] Israelachvili JN. Refinement of the fluid-mosaic model of membrane structure. Biochimica et Biophysica Acta (BBA) - Biomembranes 1977;469:221-5.

[12] Nicolson GL. The Fluid-Mosaic Model of Membrane Structure: still relevant to understanding the structure, function and dynamics of biological membranes after more than 40 years. Biochim Biophys Acta 2014;1838:1451-66.

[13] Hollmann A, Martinez M, Maturana P, Semorile LC, Maffia PC. Antimicrobial Peptides: Interaction With Model and Biological Membranes and Synergism With Chemical Antibiotics. Front Chem 2018;6:204.

[14] Hedegaard SF, Derbas MS, Lind TK, Kasimova MR, Christensen MV, Michaelsen $\mathrm{MH}$, et al. Fluorophore labeling of a cell-penetrating peptide significantly alters the mode and degree of biomembrane interaction. Sci Rep 2018;8:6327.

[15] Stenbæk J, Löf D, Falkman P, Jensen B, Cárdenas M. An alternative anionic biosustainable anti-fungal agent: Investigation of its mode of action on the fungal cell membrane. J Colloid Interface Sci 2017;497:242-8.

[16] Hancock REW, Rozek A. Role of membranes in the activities of antimicrobial cationic peptides. FEMS Microbiol Lett 2002;206:143-9.

[17] Farag MR, Alagawany M. Erythrocytes as a biological model for screening of xenobiotics toxicity. Chem Biol Interact 2018;279:73-83.

[18] Åkesson A, Lind TK, Barker R, Hughes A, Cárdenas M. Unraveling Dendrimer Translocation Across Cell Membrane Mimics. Langmuir 2012;28:13025-33. https://doi.org/10.1021/la3027144.

[19] Zhang G, Meredith TC, Kahne D. On the essentiality of lipopolysaccharide to Gramnegative bacteria. Curr Opin Microbiol 2013;16:779-85. 
[20] Nikaido H. Molecular Basis of Bacterial Outer Membrane Permeability Revisited. Microbiology and Molecular Biology Reviews 2003;67:593-656. https://doi.org/10.1128/mmbr.67.4.593-656.2003.

[21] Vollmer W, Blanot D, de Pedro MA. Peptidoglycan structure and architecture. FEMS Microbiol Rev 2008;32:149-67.

[22] Gov N, Safran SA. Red blood cell shape and fluctuations: cytoskeleton confinement and ATP activity. J Biol Phys 2005;31:453-64.

[23] Harayama T, Riezman $H$. Understanding the diversity of membrane lipid composition. Nat Rev Mol Cell Biol 2018;19:281-96.

[24] Rastogi BK, Nordøy A. Lipid composition of cultured human endothelial cells. Thromb Res 1980;18:629-41.

[25] Nordoy A, Bjorge JM, Strom E. Comparison of the main lipids in platelets and plasma in man. Acta Med Scand 1973;193:59-64.

[26] Ingólfsson HI, Carpenter TS, Bhatia H, Bremer P-T, Marrink SJ, Lightstone FC. Computational Lipidomics of the Neuronal Plasma Membrane. Biophys $\mathrm{J}$ 2017;113:2271-80.

[27] Huang J, Feigenson GW. A microscopic interaction model of maximum solubility of cholesterol in lipid bilayers. Biophys J 1999;76:2142-57.

[28] Petruzielo RS, Heberle FA, Drazba P, Katsaras J, Feigenson GW. Phase behavior and domain size in sphingomyelin-containing lipid bilayers. Biochim Biophys Acta 2013;1828:1302-13.

[29] Pomorski TG, Nylander T, Cárdenas M. Model cell membranes: discerning lipid and protein contributions in shaping the cell. Adv Colloid Interface Sci 2014;205:207-20.

[30] Pomorski T, Menon AK. Lipid flippases and their biological functions. Cell Mol Life Sci 2006;63:2908-21.

[31] Fadeel B, Xue D. The ins and outs of phospholipid asymmetry in the plasma membrane: roles in health and disease. Crit Rev Biochem Mol Biol 2009;44:264-77.

[32] Ingram LO. Changes in lipid composition of Escherichia coli resulting from growth with organic solvents and with food additives. Appl Environ Microbiol 1977;33:12336.

[33] Clejan S, Krulwich TA, Mondrus KR, Seto-Young D. Membrane lipid composition of 
obligately and facultatively alkalophilic strains of Bacillus spp. Journal of Bacteriology 1986;168:334-40. https://doi.org/10.1128/jb.168.1.334-340.1986.

[34] Sohlenkamp C, Geiger O. Bacterial membrane lipids: diversity in structures and pathways. FEMS Microbiol Rev 2016;40:133-59.

[35] Virtanen JA, Cheng KH, Somerharju P. Phospholipid composition of the mammalian red cell membrane can be rationalized by a superlattice model. Proc Natl Acad Sci U S A 1998;95:4964-9.

[36] Kaganer VM, Möhwald H, Dutta P. Structure and phase transitions in Langmuir monolayers. Rev Mod Phys 1999;71:779-819.

[37] Wiese H. A. W. Adamson: "Physical Chemistry of Surfaces", 5. Auflage, John Wiley \& Sons Inc., New York, Chichester, Brisbane, Toronto, Singapore 1990. 777 Seiten, Preis: $£$ 47.50. Berichte Der Bunsengesellschaft Für Physikalische Chemie 1991;95:758-758. https://doi.org/10.1002/bbpc.19910950629.

[38] Barzyk W, Lunkenheimer K, Warszyński P, Jachimska B, Pomianowski A. Effect of molecular structure of amphiphiles on the surface pressure and electric surface potential isotherms at the air/solution interface. Colloids Surf A Physicochem Eng Asp 2014;443:515-24.

[39] Peterson IR. A fully automated high performance Langmuir-Blodgett trough. Thin Solid Films 1985;134:135-41.

[40] Fainerman VB, Vollhardt D. Surface pressure isotherm for the fluid state of Langmuir monolayers. J Phys Chem B 2006;110:10436-40.

[41] Pfrang C, Sebastiani F, Lucas COM, King MD, Hoare ID, Chang D, et al. Ozonolysis of methyl oleate monolayers at the air-water interface: oxidation kinetics, reaction products and atmospheric implications. Phys Chem Chem Phys 2014;16:13220-8.

[42] Thompson KC, Jones SH, Rennie AR, King MD, Ward AD, Hughes BR, et al. Degradation and rearrangement of a lung surfactant lipid at the air-water interface during exposure to the pollutant gas ozone. Langmuir 2013;29:4594-602.

[43] Maget-Dana R. The monolayer technique: a potent tool for studying the interfacial properties of antimicrobial and membrane-lytic peptides and their interactions with lipid membranes. Biochimica et Biophysica Acta (BBA) - Biomembranes 1999;1462:109-40. 
[44] Elderdfi M, Sikorski AF. Langmuir-monolayer methodologies for characterizing protein-lipid interactions. Chem Phys Lipids 2018;212:61-72.

[45] Dabkowska AP, Barlow DJ, Campbell RA, Hughes AV, Quinn PJ, Lawrence MJ. Effect of helper lipids on the interaction of DNA with cationic lipid monolayers studied by specular neutron reflection. Biomacromolecules 2012;13:2391-401.

[46] Gzyl-Malcher B, Handzlik J, Klekowska E. Interaction of prazosin with model membranes--a Langmuir monolayer study. Bioelectrochemistry 2012;87:96-103.

[47] Sun H, Resmini M, Zarbakhsh A. Interaction of thermal responsive NIPAM nanogels with model lipid monolayers at the air-water interface. J Colloid Interface Sci 2018;519:97-106.

[48] Ciumac D, Campbell RA, Xu H, Clifton LA, Hughes AV, Webster JRP, et al. Implications of lipid monolayer charge characteristics on their selective interactions with a short antimicrobial peptide. Colloids Surf B Biointerfaces 2017;150:308-16.

[49] Perriman AW, McGillivray DJ, White JW. Reactions of isolated mono-molecular protein films. Soft Matter 2008;4:2192.

[50] Matyszewska D, Brzezińska K, Juhaniewicz J, Bilewicz R. pH dependence of daunorubicin interactions with model DMPC:Cholesterol membranes. Colloids Surf B Biointerfaces 2015;134:295-303.

[51] Fernandez DI, Sani M-A, Miles AJ, Wallace BA, Separovic F. Membrane defects enhance the interaction of antimicrobial peptides, aurein 1.2 versus caerin 1.1 . Biochim Biophys Acta 2013;1828:1863-72.

[52] Dimitrievski K, Kasemo B. Simulations of lipid vesicle adsorption for different lipid mixtures. Langmuir 2008;24:4077-91.

[53] Kobayashi S, Takeshima K, Park CB, Kim SC, Matsuzaki K. Interactions of the Novel Antimicrobial Peptide Buforin 2 with Lipid Bilayers: Proline as a Translocation Promoting Factor $\uparrow$. Biochemistry 2000;39:8648-54.

[54] Gragson DE, Beaman D, Porter R. Using Compression Isotherms of Phospholipid Monolayers To Explore Critical Phenomena. A Biophysical Chemistry Experiment. J Chem Educ 2008;85:272.

[55] Campbell RA, Saaka Y, Shao Y, Gerelli Y, Cubitt R, Nazaruk E, et al. Structure of surfactant and phospholipid monolayers at the air/water interface modeled from 
neutron reflectivity data. J Colloid Interface Sci 2018;531:98-108.

[56] Lind TK, Cárdenas M. Understanding the formation of supported lipid bilayers via vesicle fusion-A case that exemplifies the need for the complementary method approach (Review). Biointerphases 2016;11:020801.

[57] Cho N-J, Frank CW, Kasemo B, Höök F. Quartz crystal microbalance with dissipation monitoring of supported lipid bilayers on various substrates. Nat Protoc 2010;5:1096-106.

[58] Åkesson A, Lind T, Ehrlich N, Stamou D, Wacklin H, Cárdenas M. Composition and structure of mixed phospholipid supported bilayers formed by POPC and DPPC. Soft Matter 2012;8:5658. https://doi.org/10.1039/c2sm00013j.

[59] Lind TK, Darré L, Domene C, Urbanczyk-Lipkowska Z, Cárdenas M, Wacklin HP. Antimicrobial peptide dendrimer interacts with phosphocholine membranes in a fluidity dependent manner: A neutron reflection study combined with molecular dynamics simulations. Biochim Biophys Acta 2015;1848:2075-84.

[60] Wacklin HP. Composition and asymmetry in supported membranes formed by vesicle fusion. Langmuir 2011;27:7698-707.

[61] Lv Z, Banerjee S, Zagorski K, Lyubchenko YL. Supported Lipid Bilayers for Atomic Force Microscopy Studies. Methods Mol Biol 2018;1814:129-43.

[62] Browning KL, Lind TK, Maric S, Malekkhaiat-Häffner S, Fredrikson GN, Bengtsson E, et al. Human Lipoproteins at Model Cell Membranes: Effect of Lipoprotein Class on Lipid Exchange. Sci Rep 2017;7:7478.

[63] Stanglmaier S, Hertrich S, Fritz K, Moulin J-F, Haese-Seiller M, Rädler JO, et al. Asymmetric Distribution of Anionic Phospholipids in Supported Lipid Bilayers. Langmuir 2012;28:10818-21.

[64] Luchini A, Nzulumike ANO, Lind TK, Nylander T, Barker R, Arleth L, et al. Towards biomimics of cell membranes: Structural effect of phosphatidylinositol triphosphate (PIP) on a lipid bilayer. Colloids Surf B Biointerfaces 2019;173:202-9.

[65] Huang CH, Li S, Lin HN, Wang G. On the bilayer phase transition temperatures for monoenoic phosphatidylcholines and phosphatidylethanolamines and the interconversion between them. Arch Biochem Biophys 1996;334:135-42.

[66] Hogendorf WFJ, Jagalski V, Pomorski TG, Bols M, Cárdenas M, Pedersen CM. 
Synthesis and thermotropic phase behavior of four glycoglycerolipids. Molecules 2013;18:13546-73.

[67] Silvius JR. Solid- and liquid-phase equilibria in phosphatidylcholine/phosphatidylethanolamine mixtures. A calorimetric study. Biochim Biophys Acta 1986;857:217-28.

[68] Waldie S, Moulin M, Porcar L, Pichler H, Strohmeier GA, Skoda M, et al. The Production of Matchout-Deuterated Cholesterol and the Study of Bilayer-Cholesterol Interactions. Sci Rep 2019;9:5118.

[69] Waldie S, Lind TK, Browning K, Moulin M, Haertlein M, Forsyth VT, et al. Localization of Cholesterol within Supported Lipid Bilayers Made of a Natural Extract of TailorDeuterated Phosphatidylcholine. Langmuir 2018;34:472-9.

[70] Lawrence JC, Saslowsky DE, Edwardson JM, Henderson RM. Real-time analysis of the effects of cholesterol on lipid raft behavior using atomic force microscopy. Biophys J 2003;84:1827-32.

[71] Melby ES, Mensch AC, Lohse SE, Hu D, Orr G, Murphy CJ, et al. Formation of supported lipid bilayers containing phase-segregated domains and their interaction with gold nanoparticles. Environ Sci: Nano 2016;3:45-55.

[72] Lind TK, Wacklin H, Schiller J, Moulin M, Haertlein M, Pomorski TG, et al. Formation and Characterization of Supported Lipid Bilayers Composed of Hydrogenated and Deuterated Escherichia coli Lipids. PLoS One 2015;10:e0144671.

[73] Clifton LA, Skoda MWA, Daulton EL, Hughes AV, Le Brun AP, Lakey JH, et al. Asymmetric phospholipid: lipopolysaccharide bilayers; a Gram-negative bacterial outer membrane mimic. J R Soc Interface 2013;10:20130810.

[74] Clifton LA, Skoda MWA, Le Brun AP, Ciesielski F, Kuzmenko I, Holt SA, et al. Effect of divalent cation removal on the structure of gram-negative bacterial outer membrane models. Langmuir 2015;31:404-12.

[75] Plunkett P, Camley BA, Weirich KL, Israelachvili J, Atzberger PJ. Simulation of edge facilitated adsorption and critical concentration induced rupture of vesicles at a surface. Soft Matter 2013;9:8420.

[76] Lind TK, Cárdenas M, Wacklin HP. Formation of supported lipid bilayers by vesicle fusion: effect of deposition temperature. Langmuir 2014;30:7259-63. 
[77] Duša F, Chen W, Witos J, Wiedmer SK. Calcium Dependent Reversible Aggregation of Escherichia coli Biomimicking Vesicles Enables Formation of Supported Vesicle Layers on Silicon Dioxide. Front Mater Sci 2019;6:153.

[78] Lind TK, Skoda MWA, Cárdenas M. Formation and Characterization of Supported Lipid Bilayers Composed of Phosphatidylethanolamine and Phosphatidylglycerol by Vesicle Fusion, a Simple but Relevant Model for Bacterial Membranes. ACS Omega 2019;4:10687-94.

[79] Kurniawan J, Ventrici de Souza JF, Dang AT, Liu G-Y, Kuhl TL. Preparation and Characterization of Solid-Supported Lipid Bilayers Formed by Langmuir-Blodgett Deposition: A Tutorial. Langmuir 2018;34:15622-39.

[80] Paracini N, Clifton LA, Skoda MWA, Lakey JH. Liquid crystalline bacterial outer membranes are critical for antibiotic susceptibility. Proc Natl Acad Sci U S A 2018;115:E7587-94.

[81] Israelachvili JN, Mitchell DJ, Ninham BW. Theory of self-assembly of lipid bilayers and vesicles. Biochim Biophys Acta 1977;470:185-201.

[82] Florek OB, Clifton LA, Wilde M, Arnold T, Green RJ, Frazier RA. Lipid composition in fungal membrane models: effect of lipid fluidity. Acta Crystallogr D Struct Biol 2018;74:1233-44.

[83] Clifton LA, Ciesielski F, Skoda MWA, Paracini N, Holt SA, Lakey JH. The Effect of Lipopolysaccharide Core Oligosaccharide Size on the Electrostatic Binding of Antimicrobial Proteins to Models of the Gram Negative Bacterial Outer Membrane. Langmuir 2016;32:3485-94.

[84] Gerelli Y, Porcar L, Fragneto G. Lipid rearrangement in DSPC/DMPC bilayers: a neutron reflectometry study. Langmuir 2012;28:15922-8.

[85] Przybylo M, Sýkora J, Humpolíčková J, Benda A, Zan A, Hof M. Lipid Diffusion in Giant Unilamellar Vesicles Is More than 2 Times Faster than in Supported Phospholipid Bilayers under Identical Conditions. Langmuir 2006;22:9096-9. https://doi.org/10.1021/la061934p.

[86] Wu H-L, Tong Y, Peng Q, Li N, Ye S. Phase transition behaviors of the supported DPPC bilayer investigated by sum frequency generation (SFG) vibrational spectroscopy and atomic force microscopy (AFM). Phys Chem Chem Phys 
2016;18:1411-21.

[87] Machán R, Hof M. Lipid diffusion in planar membranes investigated by fluorescence correlation spectroscopy. Biochim Biophys Acta 2010;1798:1377-91.

[88] Tero R. Substrate Effects on the Formation Process, Structure and Physicochemical Properties of Supported Lipid Bilayers. Materials 2012;5:2658-80.

[89] Monzel C, Sengupta K. Measuring shape fluctuations in biological membranes. J Phys D Appl Phys 2016;49:243002.

[90] Alessandrini A, Facci P. Phase transitions in supported lipid bilayers studied by AFM. Soft Matter 2014;10:7145-64.

[91] Lind TK, Zielińska P, Wacklin HP, Urbańczyk-Lipkowska Z, Cárdenas M. Continuous flow atomic force microscopy imaging reveals fluidity and time-dependent interactions of antimicrobial dendrimer with model lipid membranes. ACS Nano 2014;8:396-408.

[92] Tanaka M, Sackmann E. Polymer-supported membranes as models of the cell surface. Nature 2005;437:656-63.

[93] Sackmann E. Supported membranes: scientific and practical applications. Science 1996;271:43-8.

[94] McCabe IP, Forstner MB. Polymer Supported Lipid Bilayers. OJBIPHY 2013;03:5969.

[95] Merzlyakov M, Li E, Gitsov I, Hristova K. Surface-supported bilayers with transmembrane proteins: role of the polymer cushion revisited. Langmuir 2006;22:10145-51.

[96] Diaz AJ, Albertorio F, Daniel S, Cremer PS. Double cushions preserve transmembrane protein mobility in supported bilayer systems. Langmuir 2008;24:6820-6.

[97] Jackman J, Knoll W, Cho N-J. Biotechnology Applications of Tethered Lipid Bilayer Membranes. Materials 2012;5:2637-57.

[98] Raguse B, Braach-Maksvytis V, Cornell BA, King LG, Osman PDJ, Pace RJ, et al. Tethered Lipid Bilayer Membranes: Formation and Ionic Reservoir Characterization. Langmuir 1998;14:648-59.

[99] Woodhouse GE, King LG, Wieczorek L, Cornell BA. Kinetics of the competitive 
response of receptors immobilised to ion-channels which have been incorporated into a tethered bilayer. Faraday Discuss 1999;111:247-58.

[100] Heinrich F, Ng T, Vanderah DJ, Shekhar P, Mihailescu M, Nanda H, et al. A New Lipid Anchor for Sparsely Tethered Bilayer Lipid Membranes †. Langmuir 2009;25:4219-29.

[101] Jagalski V, Barker RD, Thygesen MB, Gotfryd K, Krüger MB, Shi L, et al. Grafted biomembranes containing membrane proteins--the case of the leucine transporter. Soft Matter 2015;11:7707-11.

[102] Fragneto G, Charitat T, Daillant J. Floating lipid bilayers: models for physics and biology. Eur Biophys J 2012;41:863-74.

[103] Fragneto G, Charitat T, Bellet-Amalric E, Cubitt R, Graner F. Swelling of Phospholipid Floating Bilayers: The Effect of Chain Length $\dagger$. Langmuir 2003;19:7695-702.

[104] Hughes AV, Howse JR, Dabkowska A, Jones RAL, Lawrence MJ, Roser SJ. Floating lipid bilayers deposited on chemically grafted phosphatidylcholine surfaces. Langmuir 2008;24:1989-99.

[105] Hughes AV, Holt SA, Daulton E, Soliakov A, Charlton TR, Roser SJ, et al. High coverage fluid-phase floating lipid bilayers supported by $\omega$-thiolipid self-assembled monolayers. J R Soc Interface 2014;11:20140245.

[106] Charitat T, Lecuyer S, Fragneto G. Fluctuations and destabilization of single phospholipid bilayers. Biointerphases 2008;3:FB3.

[107] Clifton LA, Paracini N, Hughes AV, Lakey JH, Steinke N-J, Cooper JFK, et al. SelfAssembled Fluid Phase Floating Membranes with Tunable Water Interlayers. Langmuir 2019;35:13735-44.

[108] Isaksson S, Watkins EB, Browning KL, Kjellerup Lind T, Cárdenas M, Hedfalk K, et al. Protein-Containing Lipid Bilayers Intercalated with Size-Matched Mesoporous Silica Thin Films. Nano Lett 2017;17:476-85.

[109] Ebbensgaard A, Mordhorst H, Overgaard MT, Nielsen CG, Aarestrup FM, Hansen EB. Comparative Evaluation of the Antimicrobial Activity of Different Antimicrobial Peptides against a Range of Pathogenic Bacteria. PLoS One 2015;10:e0144611. [110] Juhaniewicz J, Sek S. Atomic Force Microscopy and Electrochemical Studies of 
Melittin Action on Lipid Bilayers Supported on Gold Electrodes. Electrochim Acta 2015;162:53-61.

[111] Bodescu MA, Rosenkötter F, Fritz J. Time lapse AFM on vesicle formation from mixed lipid bilayers induced by the membrane-active peptide melittin. Soft Matter 2017;13:6845-51.

[112] Rai DK, Qian S, Heller WT. The Interaction of Melittin with Dimyristoyl Phosphatidylcholine-Dimyristoyl Phosphatidylserine Lipid Bilayer Membranes. Biochim Biophys Acta 2016;1858:2788-94.

[113] Steinem C, Galla H-J, Janshoff A. Interaction of melittin with solid supported membranes. Phys Chem Chem Phys 2000;2:4580-5.

[114] Frey S, Tamm LK. Orientation of melittin in phospholipid bilayers. A polarized attenuated total reflection infrared study. Biophys J 1991;60:922-30.

[115] Lad MD, Birembaut F, Clifton LA, Frazier RA, Webster JRP, Green RJ. Antimicrobial peptide-lipid binding interactions and binding selectivity. Biophys $\mathrm{J}$ 2007;92:3575-86.

[116] Juhaniewicz J, Sek S. Interaction of Melittin with Negatively Charged Lipid Bilayers Supported on Gold Electrodes. Electrochim Acta 2016;197:336-43.

[117] Machán R, Miszta A, Hermens W, Hof M. Real-time monitoring of melittin-induced pore and tubule formation from supported lipid bilayers and its physiological relevance. Chem Phys Lipids 2010;163:200-6.

[118] Lu N, Yang K, Yuan B, Ma Y. Molecular response and cooperative behavior during the interactions of melittin with a membrane: dissipative quartz crystal microbalance experiments and simulations. J Phys Chem B 2012;116:9432-8.

[119] Irudayam SJ, Berkowitz ML. Binding and reorientation of melittin in a POPC bilayer: computer simulations. Biochim Biophys Acta 2012;1818:2975-81.

[120] Shepherd CM, Schaus KA, Vogel HJ, Juffer AH. Molecular Dynamics Study of Peptide-Bilayer Adsorption. Biophys J 2001;80:579-96.

[121] Katzir S. The Beginnings of Piezoelectricity: A Study in Mundane Physics. Springer Science \& Business Media; 2007.

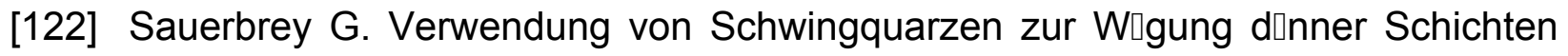


und zur Mikrow】gung. Z Physik 1959;155:206-22.

[123] Alassi A, Benammar M, Brett D. Quartz Crystal Microbalance Electronic Interfacing Systems: A Review. Sensors 2017;17. https://doi.org/10.3390/s17122799.

[124] Rodahl M, Höök F, Krozer A, Brzezinski P, Kasemo B. Quartz crystal microbalance setup for frequency and $Q$-factor measurements in gaseous and liquid environments. Review of Scientific Instruments 1995;66:3924-30. https://doi.org/10.1063/1.1145396.

[125] Höök F, Kasemo B, Nylander T, Fant C, Sott K, Elwing H. Variations in Coupled Water, Viscoelastic Properties, and Film Thickness of a Mefp-1 Protein Film during Adsorption and Cross-Linking: A Quartz Crystal Microbalance with Dissipation Monitoring, Ellipsometry, and Surface Plasmon Resonance Study. Analytical Chemistry 2001;73:5796-804. https://doi.org/10.1021/ac0106501.

[126] Parsons R. Ellipsometry, proceeding of the third international conference on ellipsometry Edited by N.M. Bashara and R.M.A. Azzam, North Holland, Amsterdam, 1976, 518 pp., price Dfl. 190. Journal of Electroanalytical Chemistry 1977;79:201-2. https://doi.org/10.1016/0368-1874(77)80216-5.

[127] van Oss CJ. Physical Chemistry or Biological Interfaces A. Baszkin \& W. Norde, editors. Marcel Dekker, Inc. New York, 1999, hardbound, pp. ix 836; \$225. Journal of Dispersion Science and Technology 2000;21:365-6. https://doi.org/10.1080/01932690008913272.

[128] De Feijter JA, Benjamins J, Veer FA. Ellipsometry as a tool to study the adsorption behavior of synthetic and biopolymers at the air-water interface. Biopolymers 1978;17:1759-72.

[129] Cuypers PA, Corsel JW, Janssen MP, Kop JMM, Hermens WT, Hemker HC. The adsorption of prothrombin to phosphatidylserine multilayers quantitated by ellipsometry. J Biol Chem 1983;258:2426-2431.

[130] Landgren M, Joensson B. Determination of the optical properties of silicon/silica surfaces by means of ellipsometry, using different ambient media. J Phys Chem 1993;97:1656-60.

[131] Tiberg F, Landgren M. Characterization of thin nonionic surfactant films at the silica/water interface by means of ellipsometry. Langmuir 1993;9:927-32. 
[132] Langevin D. Light Scattering by Liquid Surfaces and Complementary Techniques. Taylor \& Francis; 1991.

[133] Howland MC, Szmodis AW, Sanii B, Parikh AN. Characterization of physical properties of supported phospholipid membranes using imaging ellipsometry at optical wavelengths. Biophys J 2007;92:1306-17.

[134] Tiberg F, Harwigsson I, Malmsten M. Formation of model lipid bilayers at the silicawater interface by co-adsorption with non-ionic dodecyl maltoside surfactant. Eur Biophys J 2000;29:196-203.

[135] Deleu M, Lorent J, Lins L, Brasseur R, Braun N, El Kirat K, et al. Effects of surfactin on membrane models displaying lipid phase separation. Biochim Biophys Acta 2013;1828:801-15.

[136] Deleu M, Paquot M, Nylander T. Effect of fengycin, a lipopeptide produced by Bacillus subtilis, on model biomembranes. Biophys J 2008;94:2667-79.

[137] Ringstad L, Andersson Nordahl E, Schmidtchen A, Malmsten M. Composition effect on peptide interaction with lipids and bacteria: variants of C3a peptide CNY21. Biophys J 2007;92:87-98.

[138] Milyaeva OY, Gochev G, Loglio G, Miller R, Noskov BA. Influence of polyelectrolytes on dynamic surface properties of fibrinogen solutions. Colloids Surf A Physicochem Eng Asp 2017;532:108-15.

[139] Angus-Smyth A, Campbell RA, Bain CD. Dynamic adsorption of weakly interacting polymer/surfactant mixtures at the air/water interface. Langmuir 2012;28:12479-92.

[140] Benjamins J-W, Thuresson K, Nylander T. Ellipsometry studies of nonionic surfactant adsorption at the oil--water interface. Langmuir 2005;21:149-59.

[141] Thoma M, Schwendler M, Baltes H, Helm CA, Pfohl T, Riegler H, et al. Ellipsometry and X-ray Reflectivity Studies on Monolayers of Phosphatidylethanolamine and Phosphatidylcholine in Contact with $\mathrm{n}$-Dodecane, $\mathrm{n}$-Hexadecane, and Bicyclohexyl. Langmuir 1996;12:1722-8.

[142] Miñones J Jr, Rodríguez Patino JM, Conde O, Carrera C, Seoane R. The effect of polar groups on structural characteristics of phospholipid monolayers spread at the air-water interface. Colloids Surf A Physicochem Eng Asp 2002;203:273-86.

[143] Velasco E, Mederos L. Anisotropic line tension of domains in lipid monolayers. 
Phys Rev E 2019;100:032413.

[144] Tummino A, Toscano J, Sebastiani F, Noskov BA, Varga I, Campbell RA. Effects of Aggregate Charge and Subphase Ionic Strength on the Properties of Spread Polyelectrolyte/Surfactant Films at the Air/Water Interface under Static and Dynamic Conditions. Langmuir 2018;34:2312-23.

[145] Campbell RA, Yanez Arteta M, Angus-Smyth A, Nylander T, Noskov BA, Varga I. Direct impact of nonequilibrium aggregates on the structure and morphology of Pdadmac/SDS layers at the air/water interface. Langmuir 2014;30:8664-74.

[146] Garcia-Manyes S, Domènech O, Sanz F, Montero MT, Hernandez-Borrell J. Atomic force microscopy and force spectroscopy study of Langmuir-Blodgett films formed by heteroacid phospholipids of biological interest. Biochim Biophys Acta 2007;1768:1190-8.

[147] Binnig G, Quate CF, Gerber C. Atomic force microscope. Phys Rev Lett 1986;56:930-3.

[148] García R. Dynamic atomic force microscopy methods. Surf Sci Rep 2002;47:197301.

[149] Dufrêne YF, Ando T, Garcia R, Alsteens D, Martinez-Martin D, Engel A, et al. Imaging modes of atomic force microscopy for application in molecular and cell biology. Nat Nanotechnol 2017;12:295-307.

[150] Butt H-J, Cappella B, Kappl M. Force measurements with the atomic force microscope: Technique, interpretation and applications. Surf Sci Rep 2005;59:1152.

[151] Dufrêne YF, Lee GU. Advances in the characterization of supported lipid films with the atomic force microscope. Biochimica et Biophysica Acta (BBA) - Biomembranes 2000;1509:14-41.

[152] Dufrêne YF, Martínez-Martín D, Medalsy I, Alsteens D, Müller DJ. Multiparametric imaging of biological systems by force-distance curve-based AFM. Nat Methods 2013;10:847-54.

[153] Garcia-Manyes S, Sanz F. Nanomechanics of lipid bilayers by force spectroscopy with AFM: a perspective. Biochim Biophys Acta 2010;1798:741-9.

[154] Muller DJ. AFM: a nanotool in membrane biology. Biochemistry 2008;47:7986-98. 
[155] Butt H-J, -J Butt H, Jaschke M. Calculation of thermal noise in atomic force microscopy. Nanotechnology 1995;6:1-7. https://doi.org/10.1088/09574484/6/1/001.

[156] Sader JE, Chon JWM, Mulvaney P. Calibration of rectangular atomic force microscope cantilevers. Rev Sci Instrum 1999;70:3967-9.

[157] Das C, Sheikh KH, Olmsted PD, Connell SD. Nanoscale mechanical probing of supported lipid bilayers with atomic force microscopy. Phys Rev E Stat Nonlin Soft Matter Phys 2010;82:041920.

[158] Dufrêne YF, Barger WR, Green J-BD, Lee GU. Nanometer-Scale Surface Properties of Mixed Phospholipid Monolayers and Bilayers. Langmuir 1997;13:4779_ 84.

[159] Garcia-Manyes S, Oncins G, Sanz F. Effect of temperature on the nanomechanics of lipid bilayers studied by force spectroscopy. Biophys J 2005;89:4261-74.

[160] Garcia-Manyes S, Oncins G, Sanz F. Effect of ion-binding and chemical phospholipid structure on the nanomechanics of lipid bilayers studied by force spectroscopy. Biophys J 2005;89:1812-26.

[161] Leonenko ZV, Finot E, Ma H, Dahms TES, Cramb DT. Investigation of temperature-induced phase transitions in DOPC and DPPC phospholipid bilayers using temperature-controlled scanning force microscopy. Biophys J 2004;86:378393.

[162] Picas L, Rico F, Scheuring S. Direct measurement of the mechanical properties of lipid phases in supported bilayers. Biophys J 2012;102:L01-3.

[163] An H, Nussio MR, Huson MG, Voelcker NH, Shapter JG. Material properties of lipid microdomains: force-volume imaging study of the effect of cholesterol on lipid microdomain rigidity. Biophys J 2010;99:834-44.

[164] Medalsy I, Hensen U, Muller DJ. Imaging and quantifying chemical and physical properties of native proteins at molecular resolution by force-volume AFM. Angew Chem Int Ed Engl 2011;50:12103-8.

[165] Palacios-Lidón E, Munuera C, Ocal C, Colchero J. Contrast inversion in noncontact Dynamic Scanning Force Microscopy: what is high and what is low? Ultramicroscopy 2010;110:789-800. 
[166] Egger M, Ohnesorge F, Weisenhorn AL, Heyn SP, Drake B, Prater CB, et al. Wet lipid-protein membranes imaged at submolecular resolution by atomic force microscopy. J Struct Biol 1990;103:89-94.

[167] Hansma HG, Gould SAC, Hansma PK, Gaub HE, Longo ML, Zasadzinski JAN. Imaging nanometer scale defects in Langmuir-Blodgett films with the atomic force microscope. Langmuir 1991;7:1051-4.

[168] Chi LF, Anders M, Fuchs H, Johnston RR, Ringsdorf H. Domain structures in langmuir-blodgett films investigated by atomic force microscopy. Science 1993;259:213-6.

[169] Reviakine I, Brisson A. Formation of Supported Phospholipid Bilayers from Unilamellar Vesicles Investigated by Atomic Force Microscopy. Langmuir 2000;16:1806-15.

[170] Mou J, Yang J, Huang C, Shao Z. Alcohol Induces Interdigitated Domains in Unilamellar Phosphatidylcholine Bilayers. Biochemistry 1994;33:9981-5.

[171] Rinia HA, Kik RA, Demel RA, Snel MME, Killian JA, van der Eerden JPJM, et al. Visualization of Highly Ordered Striated Domains Induced by Transmembrane Peptides in Supported Phosphatidylcholine Bilayers †. Biochemistry 2000;39:58528.

[172] Yarrow F, Kuipers BWM. AFM study of the thermotropic behaviour of supported DPPC bilayers with and without the model peptide WALP23. Chem Phys Lipids 2011;164:9-15.

[173] Hénon S, Meunier J. Microscope at the Brewster angle: Direct observation of first-order phase transitions in monolayers. Review of Scientific Instruments 1991;62:936-9. https://doi.org/10.1063/1.1142032.

[174] Roldán-Carmona C, Giner-Casares JJ, Pérez-Morales M, Martín-Romero MT, Camacho L. Revisiting the Brewster Angle Microscopy: the relevance of the polar headgroup. Adv Colloid Interface Sci 2012;173:12-22.

[175] Daear W, Mahadeo M, Prenner EJ. Applications of Brewster angle microscopy from biological materials to biological systems. Biochim Biophys Acta Biomembr 2017;1859:1749-66.

[176] Wydro P, Flasiński M, Broniatowski M. Molecular organization of bacterial 
membrane lipids in mixed systems-A comprehensive monolayer study combined with Grazing Incidence X-ray Diffraction and Brewster Angle Microscopy experiments. Biochimica et Biophysica Acta (BBA) - Biomembranes 2012;1818:1745-54. https://doi.org/10.1016/j.bbamem.2012.03.010.

[177] Castano S, Desbat B. Structure and orientation study of fusion peptide FP23 of gp41 from HIV-1 alone or inserted into various lipid membrane models (mono-, biand multibi-layers) by FT-IR spectroscopies and Brewster angle microscopy. Biochim Biophys Acta 2005;1715:81-95.

[178] Foglia F, Fragneto G, Clifton LA, Lawrence MJ, Barlow DJ. Interaction of amphotericin B with lipid monolayers. Langmuir 2014;30:9147-56.

[179] Clifton LA, Sanders M, Kinane C, Arnold T, Edler KJ, Neylon C, et al. The role of protein hydrophobicity in thionin-phospholipid interactions: a comparison of $\alpha 1$ and a2-purothionin adsorbed anionic phospholipid monolayers. Physical Chemistry Chemical Physics 2012;14:13569. https://doi.org/10.1039/c2cp42029e.

[180] Alvares DS, Wilke N, Ruggiero Neto J, Fanani ML. The insertion of Polybia-MP1 peptide into phospholipid monolayers is regulated by its anionic nature and phase state. Chem Phys Lipids 2017;207:38-48.

[181] Tatulian SA. Attenuated total reflection Fourier transform infrared spectroscopy: a method of choice for studying membrane proteins and lipids. Biochemistry 2003;42:11898-907.

[182] Tatulian SA. Structural Characterization of Membrane Proteins and Peptides by FTIR and ATR-FTIR Spectroscopy. Methods in Molecular Biology 2013:177-218. https://doi.org/10.1007/978-1-62703-275-9_9.

[183] Stuart BH, Ando DJ. Biological Applications of Infrared Spectroscopy. John Wiley \& Sons; 1997.

[184] Mendelsohn R, Mao G, Flach CR. Infrared reflection-absorption spectroscopy: principles and applications to lipid-protein interaction in Langmuir films. Biochim Biophys Acta 2010;1798:788-800.

[185] Wang L, Brauner JW, Mao G, Crouch E, Seaton B, Head J, et al. Interaction of recombinant surfactant protein $D$ with lipopolysaccharide: conformation and orientation of bound protein by IRRAS and simulations. Biochemistry 2008;47:8103- 
13.

[186] Dabkowska AP, Barlow DJ, Clifton LA, Hughes AV, Webster JRP, Green RJ, et al. Calcium-mediated binding of DNA to 1,2-distearoyl-sn-glycero-3-phosphocholinecontaining mixed lipid monolayers. Soft Matter 2014;10:1685-95.

[187] Clifton LA, Hall SCL, Mahmoudi N, Knowles TJ, Heinrich F, Lakey JH. Structural Investigations of Protein-Lipid Complexes Using Neutron Scattering. Methods in Molecular Biology 2019:201-51. https://doi.org/10.1007/978-1-4939-9512-7_11.

[188] Sivia DS. Elementary Scattering 2011. https://doi.org/10.1093/acprof:oso/9780199228676.001.0001.

[189] Wacklin HP. Neutron reflection from supported lipid membranes. Current Opinion in Colloid \& Interface Science 2010;15:445-54. https://doi.org/10.1016/j.cocis.2010.05.008.

[190] Nielsen JE, Lind TK, Lone A, Gerelli Y, Hansen PR, Jenssen H, et al. A biophysical study of the interactions between the antimicrobial peptide indolicidin and lipid model systems. Biochimica et Biophysica Acta (BBA) - Biomembranes 2019;1861:135564. https://doi.org/10.1016/j.bbamem.2019.04.003.

[191] Heinrich F, Lösche M. Zooming in on disordered systems: Neutron reflection studies of proteins associated with fluid membranes. Biochimica et Biophysica Acta (BBA) Biomembranes 2014;1838:2341-9. https://doi.org/10.1016/j.bbamem.2014.03.007.

[192] Braun L, Uhlig M, von Klitzing R, Campbell RA. Polymers and surfactants at fluid interfaces studied with specular neutron reflectometry. Adv Colloid Interface Sci 2017;247:130-48.

[193] Campbell RA, Tummino A, Noskov BA, Varga I. Polyelectrolyte/surfactant films spread from neutral aggregates. Soft Matter 2016;12:5304-12.

[194] Ciumac D, Campbell RA, Clifton LA, Xu H, Fragneto G, Lu JR. Influence of Acyl Chain Saturation on the Membrane-Binding Activity of a Short Antimicrobial Peptide. ACS Omega 2017;2:7482-92.

[195] Watkins EB, Miller CE, Mulder DJ, Kuhl TL, Majewski J. Structure and orientational texture of self-organizing lipid bilayers. Phys Rev Lett 2009;102:238101.

[196] Skoda MWA. Recent developments in the application of X-ray and neutron 
reflectivity to soft matter systems. Curr Opin Colloid Interface Sci 2019. https://doi.org/10.1016/j.cocis.2019.03.003.

[197] Lu JR, Thomas RK, Penfold J. Surfactant layers at the air/water interface: structure and composition. Advances in Colloid and Interface Science 2000;84:143-304. https://doi.org/10.1016/s0001-8686(99)00019-6.

[198] Stefaniu C, Brezesinski G. X-ray investigation of monolayers formed at the soft air/water interface. Curr Opin Colloid Interface Sci 2014;19:216-27.

[199] Thomas RK, Penfold J. Neutron and X-ray reflectometry of interfacial systems in colloid and polymer chemistry. Curr Opin Colloid Interface Sci 1996;1:23-33.

[200] Travkova OG, Moehwald H, Brezesinski G. The interaction of antimicrobial peptides with membranes. Adv Colloid Interface Sci 2017;247:521-32.

[201] Stefaniu C, Brezesinski G. X-ray investigation of monolayers formed at the soft air/water interface. Curr Opin Colloid Interface Sci 2014;19:216-27.

[202] Brezesinski G, Schneck E. Investigating lons at Amphiphilic Monolayers with Xray Fluorescence. Langmuir 2019;35:8531-42.

[203] Principles and applications of grazing incidence X-ray and neutron scattering from ordered molecular monolayers at the air-water interface. Phys Rep 1994;246:251313.

[204] Raghuraman H, Chattopadhyay A. Melittin: a membrane-active peptide with diverse functions. Biosci Rep 2007;27:189-223.

[205] Bach D, Miller IR. Hydration of phospholipid bilayers in the presence and absence of cholesterol. Chem Phys Lipids 2005;136:67-72.

[206] Zhang YP, Lewis RN, McElhaney RN. Calorimetric and spectroscopic studies of the thermotropic phase behavior of the n-saturated 1,2-diacylphosphatidylglycerols. Biophys J 1997;72:779-93.

[207] Wadsäter M, Simonsen JB, Lauridsen T, Tveten EG, Naur P, Bjørnholm T, et al. Aligning nanodiscs at the air-water interface, a neutron reflectivity study. Langmuir 2011;27:15065-73.

[208] Ulrich AS, Sami M, Watts A. Hydration of DOPC bilayers by differential scanning calorimetry. Biochim Biophys Acta 1994;1191:225-30.

[209] Biltonen RL, Lichtenberg D. The use of differential scanning calorimetry as a tool 
to characterize liposome preparations. Chemistry and Physics of Lipids 1993;64:129-42. https://doi.org/10.1016/0009-3084(93)90062-8. 